\title{
NONCOMMUTATIVE MOTIVES OF AZUMAYA ALGEBRAS
}

\author{
GONÇALO TABUADA AND MICHEL VAN DEN BERGH
}

\begin{abstract}
Let $k$ be a base commutative ring, $R$ a commutative ring of coefficients, $X$ a quasi-compact quasi-separated $k$-scheme with $m$ connected components, $A$ a sheaf of Azumaya algebras over $X$ of $\operatorname{rank}\left(r_{1}, \ldots, r_{m}\right)$, and $\mathrm{Hmo}_{0}(k)_{R}$ the category of noncommutative motives with $R$-coefficients. Assume that $1 / r \in R$ with $r:=r_{1} \times \cdots \times r_{m}$. Under these assumptions, we prove that the noncommutative motives with $R$-coefficients of $X$ and $A$ are isomorphic. As an application, we show that all the $R$-linear additive invariants of $X$ and $A$ are exactly the same. Examples include (nonconnective) algebraic $K$-theory, cyclic homology (and all its variants), topological Hochschild homology, etc. Making use of these isomorphisms, we then compute the $R$-linear additive invariants of differential operators in positive characteristic, of cubic fourfolds containing a plane, of Severi-Brauer varieties, of Clifford algebras, of quadrics, and of finite dimensional $k$-algebras of finite global dimension. Along the way we establish two results of independent interest. The first one asserts that every element $\alpha \in K_{0}(X)$ of rank $\left(r_{1}, \ldots, r_{m}\right)$ becomes invertible in the $R$-linearized Grothendieck group $K_{0}(X)_{R}$, and the second one that every additive invariant of finite dimensional algebras of finite global dimension is unaffected under nilpotent extensions.
\end{abstract}

\section{INTRODUCTION}

Azumaya algebras. Sheaves of Azumaya algebras over schemes $X$ were introduced in the late sixties by Grothendieck [15]. Formally, a sheaf $A$ of $\mathcal{O}_{X}$-algebras is Azumaya if it is locally free of finite rank over $\mathcal{O}_{X}$ and the canonical morphism

$$
A^{\mathrm{op}} \otimes_{\mathcal{O}_{X}} A \stackrel{\sim}{\longrightarrow} \operatorname{Hom}_{\mathcal{O}_{X}}(A, A)
$$

is an isomorphism. Locally, for the étale topology, $A$ is simply a matrix algebra. This generalizes the notion of an Azumaya algebra over a commutative ring [2, 3] and consequently the notion of a central simple algebra over a field.

Noncommutative motives. A differential graded $(=d g)$ category $\mathcal{A}$, over a base commutative ring $k$, is a category enriched over complexes of $k$-modules; see $\S 4$. Every (dg) $k$-algebra $S$ gives rise to a dg category $\underline{S}$ with a single object and (dg) $k$-algebra of endomorphisms $S$. In the same vein, every quasi-compact quasiseparated $k$-scheme $X$ gives rise to a (canonical) dg category perf ${ }_{\mathrm{dg}}(X)$ which enhances the derived category perf $(X)$ of perfect complexes of $\mathcal{O}_{X}$-modules; consult $\S 6$ for details. Let us denote by $\operatorname{dgcat}(k)$ the category of (small) dg categories.

Date: September 2, 2013.

2000 Mathematics Subject Classification. 14A22, 14F05, 16H05, 18D20, 19D55, $19 \mathrm{E} 08$.

Key words and phrases. Azumaya algebras, noncommutative motives, nilinvariance, algebraic $K$-theory, cyclic homology, noncommutative algebraic geometry.

G. Tabuada was partially supported by the NEC Award-2742738.

M. Van den Bergh is a Director of Research at the FWO Flanders.

This material is based upon work supported by the National Science Foundation (NSF) under the Grant No. 0932078 000, while the authors were in residence at the Mathematical Science Research Institute (MSRI) in Berkeley, California, during the Spring semester of 2013. 
Classical invariants such as algebraic $K$-theory $(K)$, nonconnective algebraic $K$ theory $(\mathbb{K})$, Hochschild homology $(H H)$, cyclic homology $(H C)$, periodic cyclic homology $(H P)$, negative cyclic homology $(H N)$, topological Hochschild homology $(T H H)$, and topological cyclic homology $(T C)$, extend naturally from $k$-algebras to dg categories. In order to study all these invariants simultaneously the notion of additive invariant was introduced in [37]. Let us now recall it. Given a dg category $\mathcal{A}$, let $T(\mathcal{A})$ be the dg category of pairs $(i, x)$, where $i \in\{1,2\}$ and $x$ is an object of $\mathcal{A}$. The complex of morphisms in $T(\mathcal{A})$ from $(i, x)$ to $\left(i^{\prime}, x^{\prime}\right)$ is given by $\mathcal{A}\left(x, x^{\prime}\right)$ if $i^{\prime} \geq i$ and is zero otherwise. Composition is induced by $\mathcal{A}$; consult $[37, \S 4]$ for details. Intuitively speaking, $T(\mathcal{A})$ "dg categorifies" the notion of upper triangular matrix. Note that we have two inclusion dg functors $i_{1}: \mathcal{A} \hookrightarrow T(\mathcal{A})$ and $i_{2}: \mathcal{A} \hookrightarrow T(\mathcal{A})$. A functor $E: \operatorname{dgcat}(k) \rightarrow \mathrm{D}$, with values in an additive category, is called an additive invariant if it satisfies the following two conditions:

(i) it sends Morita equivalences (see §4) to isomorphisms;

(ii) given a $\mathrm{dg}$ category $\mathcal{A}$, the inclusion dg functors induce an isomorphism ${ }^{1}$

$$
\left[E\left(i_{1}\right) E\left(i_{2}\right)\right]: E(\mathcal{A}) \oplus E(\mathcal{A}) \stackrel{\sim}{\longrightarrow} E(T(\mathcal{A})) .
$$

Thanks to the work of Blumberg-Mandell, Keller, Quillen, Schlichting, ThomasonTrobaugh, Waldhausen, and others (see [7, 18, 19, 31, 33, 35, 39, 41]), all the above invariants are additive. Moreover, when applied to $\underline{S}$, resp. to $\operatorname{perf}_{\mathrm{dg}}(X)$, they agree with the classical invariants of (dg) $k$-algebras, resp. of $k$-schemes.

Let $R$ be a commutative ring of coefficients. In [37] the universal additive invariant (with $R$-coefficients) was constructed

$$
U(-)_{R}: \operatorname{dgcat}(k) \longrightarrow \mathrm{Hmo}_{0}(k)_{R} .
$$

Given any $R$-linear additive category $\mathrm{D}$, there is an induced equivalence of categories

$$
U(-)_{R}^{*}: \operatorname{Fun}_{R \text {-linear }}\left(\mathrm{Hmo}_{0}(k)_{R}, \mathrm{D}\right) \stackrel{\sim}{\longrightarrow} \operatorname{Fun}_{\text {Additive }}(\operatorname{dgcat}(k), \mathrm{D}),
$$

where the left-hand-side denotes the category of additive $R$-linear functors and the right-hand-side the category of additive invariants. Because of this universal property, which is reminiscent from motives, $\operatorname{Hmo}_{0}(k)_{R}$ is called the category of noncommutative motives (with $R$-coefficients); consult $\S 5$ for further details. The tensor product of $k$-algebras extends also naturally to dg categories, giving thus rise to a symmetric monoidal structure $-\otimes-$ on $\operatorname{dgcat}(k)$. After deriving it, this structure descends to $\mathrm{Hmo}_{0}(k)_{R}$ and makes the functor (1.1) symmetric monoidal.

Motivation. Let $X$ be a quasi-compact quasi-separated $k$-scheme and $A$ a sheaf of Azumaya algebras over $X$. Similarly to $\operatorname{perf}_{\mathrm{dg}}(X)$, one can construct the $\mathrm{dg}$ category $\operatorname{perf}_{\mathrm{dg}}(A)$ of perfect complexes of $A$-modules; see $\S 6$. This dg category reduces to $\operatorname{perf}_{\mathrm{dg}}(X)$ when $A=\mathcal{O}_{X}$ and comes equipped with a canonical $\mathrm{dg}$ functor $-\otimes_{\mathcal{O}_{X}} A: \operatorname{perf}_{\mathrm{dg}}(X) \rightarrow \operatorname{perf}_{\mathrm{dg}}(A)$. One obtains in this way two welldefined noncommutative motives

$$
U\left(\operatorname{perf}_{\mathrm{dg}}(X)\right)_{R} \quad U\left(\operatorname{perf}_{\mathrm{dg}}(A)\right)_{R} .
$$

As mentioned above, $A$ is étale-locally a matrix algebra. Hence, up to an étale covering of $X, A$ and $\mathcal{O}_{X}$ are Morita equivalent. This leads naturally to the following motivating question: How "close" are the above noncommutative motives (1.3) ?

\footnotetext{
${ }^{1}$ Condition (ii) can be equivalently formulated in terms of semi-orthogonal decompositions in the sense of Bondal-Orlov [8]; see [37, Thm. 6.3(4)].
} 
In this article we provide a precise and complete answer to this question. As a by-product we obtain several applications of general interest; see $\S 3$.

\section{Statement of RESUlts}

Let $k$ be a base commutative ring and $R$ a commutative ring of coefficients. Recall that a scheme $X$ is quasi-compact if it admits a finite covering by affine open subschemes, and quasi-separated if the intersection of any two affine open subschemes is quasi-compact. Note that every such scheme $X$ has always a finite number of connected components. Our main result, which answers the above motivating question, is the following:

Theorem 2.1. Let $X$ be a quasi-compact quasi-separated $k$-scheme with $m$ connected components, and $A$ a sheaf of Azumaya algebras over $X$ of rank $\left(r_{1}, \ldots, r_{m}\right)$. Assume that $1 / r \in R$ with $r:=r_{1} \times \cdots \times r_{m}$. Under these assumptions, one has the following isomorphism

$$
U\left(-\otimes_{\mathcal{O}_{X}} A\right)_{R}: U\left(\operatorname{perf}_{\mathrm{dg}}(X)\right)_{R} \stackrel{\sim}{\longrightarrow} U\left(\operatorname{perf}_{\mathrm{dg}}(A)\right)_{R} .
$$

Theorem 2.1 shows us that the difference between the noncommutative motives (1.3) is simply a $r$-torsion phenomenon. As explained in Remark 3.7 below, this result is optimal, i.e. it does not hold without the assumption $1 / r \in R$.

In order to prove Theorem 2.1 we have established a $K$-theoretical result, which is of independent interest. Recall from [42, page 71] that given a scheme $X$ with $m$ connected components, one has a well-defined (split surjective) ring homomorphism rank : $K_{0}(X) \rightarrow \mathbb{Z}^{m}$. Let us write $I_{X}$ for its kernel. Whenever $X$ is Noetherian ${ }^{2}$, of Krull dimension $d$, and admits an ample sheaf, we have $I_{X}^{d+1}=0$; see $[13, \S \mathrm{V}$ Cor. 3.10]. If one does not require a uniform bound on the order of nilpotency of the elements in $I_{X}$ then this result may be generalized as follows:

Theorem 2.3. Let $X$ be a quasi-compact quasi-separated scheme $X$. Under this assumption, every element in $K_{0}(X)$ of rank zero is nilpotent.

This statement appears not to exist in the literature. The affine case was proved by Gabber in [14, page 188] using absolute noetherian approximation. ${ }^{3}$

Making use of Theorem 2.3, one obtains the following useful invertibility result:

Corollary 2.4. Let $X$ be as in Theorem 2.3 and $\alpha$ an element in $K_{0}(X)$ of rank $\left(r_{1}, \ldots, r_{m}\right)$. Assume that $1 / r \in R$ with $r:=r_{1} \times \cdots \times r_{m}$. Under these assumptions, the image of $\alpha$ in $K_{0}(X)_{R}$ is invertible.

\section{Applications}

Additive invariants. Let $k$ be a base commutative ring. As explained above, all the classical invariants of quasi-compact quasi-separated $k$-schemes $X$ can be recovered from the $\mathrm{dg}$ category $\operatorname{perf}_{\mathrm{dg}}(X)$. Hence, given a sheaf $A$ of Azumaya algebras over $X$ and an additive invariant $E: \operatorname{dgcat}(k) \rightarrow \mathrm{D}$, let us write $E(A)$ for the value of $E$ at $\operatorname{perf}_{\mathrm{dg}}(A)$. By combining Theorem 2.1 with the above equivalence (1.2) of categories one obtains the following result:

\footnotetext{
${ }^{2}$ Note that every noetherian scheme is quasi-compact and quasi-separated.

${ }^{3}$ Ben Antieau [1] has indicated to us how Gabber's approach can be extended to the general case by using absolute noetherian approximation for quasi-compact quasi-separated schemes and the local global spectral sequence for nonconnective K-theory. Our argument uses simply MayerVietoris and does not depend on absolute noetherian approximation.
} 
Corollary 3.1. Let $X, A, r, R$ be as in Theorem 2.1, and $E: \operatorname{dgcat}(k) \rightarrow \mathrm{D}$ an additive invariant with values in a R-linear category. Under these assumptions, one has an isomorphism $E(X) \simeq E(A)$.

When applied to the above examples of additive invariants, Corollary 3.1 gives rise to the following (concrete) isomorphisms

$$
\begin{array}{rlrl}
K_{*}(X)_{1 / r} \simeq K_{*}(A)_{1 / r} & \mathbb{K}_{*}(X)_{1 / r} \simeq \mathbb{K}_{*}(A)_{1 / r} \\
T H H_{*}(X)_{1 / r} \simeq T H H_{*}(A)_{1 / r} & T C_{*}(X)_{1 / r} \simeq T C_{*}(A)_{1 / r},
\end{array}
$$

where $(-)_{1 / r}:=(-)_{\mathbb{Z}[1 / r]}$. When $1 / r \in k$ one has moreover the isomorphisms

$$
\begin{array}{rlrl}
H H_{*}(X) & \simeq H H_{*}(A) & H C_{*}(X) \simeq H C_{*}(A) \\
H P_{*}(X) \simeq H P_{*}(A) & H N_{*}(X) \simeq H N_{*}(A) .
\end{array}
$$

Remark 3.6. By considering $K, \mathbb{K}, T H H$ and $T C$ as spectra-valued functors one observes that the isomorphisms (3.2)-(3.3) can be lifted to the homotopy category of spectra localized at the $\mathbb{Z}[1 / r]$-linear stable equivalences. In the same vein, the isomorphisms (3.4)-(3.5) admit a lifting to the derived category of mixed complexes; consult Keller's survey [17, §5.3] for details

The isomorphism $H H_{*}(X) \simeq H H_{*}(A)$ is well-known and holds without the assumption $1 / r \in k$. In what concerns cyclic homology, the isomorphism $H C_{*}(X) \simeq$ $H C_{*}(A)$ was established by Cortiñas-Weibel [12] in the affine case. The algebraic $K$-theory isomorphism $K_{*}(X)_{1 / r} \simeq K_{*}(A)_{1 / r}$ was obtained recently by HazratHoobler [16] under the assumption that $X$ is either regular noetherian or noetherian of finite Krull dimension with an ample sheaf. Besides these particular cases, all the remaining isomorphisms provided by Corollary 3.1 are, to the best of the authors knowledge, new in the literature.

Remark 3.7. The above isomorphism (2.2) is optimal, i.e. it does not hold without the assumption $1 / r \in R$. An example is given by the quartenions $\mathbb{H}$, considered as a central simple $\mathbb{R}$-algebra of dimension 4 . Recall from [42, page 181] that $K_{1}(\mathbb{R}) \simeq \mathbb{R}^{\times}$and $K_{1}(\mathbb{H}) \simeq \mathbb{R}_{+}^{\times}$. These groups are not (abstractly) isomorphic since $\mathbb{R}^{\times} \simeq \mathbb{R}_{+}^{\times} \times \mathbb{Z} / 2 \mathbb{Z}$ has 2 -torsion while $\mathbb{R}_{+}^{\times}$is torsion-free. Using Corollary 3.1 one then concludes that $U(\underline{\mathbb{R}})_{R} \neq U(\underline{\mathbb{H}})_{R}$ whenever $1 / 2 \notin R$. Instead of $K_{1}$ one may also use other higher $K$-groups to obtain counterexamples. Indeed, as explained in [42, page 475$], K_{2}(\mathbb{R})$ has 2-torsion and $K_{2}(\mathbb{H})$ is torsion-free, and $K_{5}(\mathbb{R}), K_{6}(\mathbb{R})$ are torsion-free and $K_{5}(\mathbb{H}), K_{6}(\mathbb{H})$ have 2-torsion.

Differential operators in positive characteristic. Let $k$ be an algebraically closed field of characteristic $p>0, X$ a smooth $k$-scheme ${ }^{4}, T^{*} X^{(1)}$ the Frobenius twist of the total cotangent bundle of $X$, and $\mathcal{D}_{X}$ the sheaf of (crystalline) differential operators on $X$; consult $[6, \S 1]$ for details. As proved by BezrukavnikovMirković-Rumynin in [6, Thm. 2.2.3], $\mathcal{D}_{X}$ is a sheaf of Azumaya algebras over $T^{*} X^{(1)}$ of rank $p^{2 \operatorname{dim}(X)}$. In the particular case where $X$ is affine space $\mathbb{A}^{n}:=$ $\operatorname{Spec}\left(k\left[x_{1}, \ldots, x_{n}\right]\right), \mathcal{D}_{X}$ reduces to the Weyl algebra $\left(\partial_{i}:=\partial / \partial x_{i}\right)$

$$
k\left\langle x_{1}, \ldots, x_{n}, \partial_{1}, \ldots, \partial_{n}\right\rangle \quad\left[\partial_{i}, x_{j}\right]=\delta_{i j}
$$

and $T^{*} X^{(1)}$ to polynomials in $2 n$ variables $k\left[x_{1}^{p}, \ldots, x_{n}^{p}, \partial_{1}^{p}, \ldots, \partial_{n}^{p}\right]$; consult $[6$, page 951 ] as well as Revoy's work [32]. Thanks to Theorem 2.1 (with $X=T^{*} X^{(1)}$

\footnotetext{
${ }^{4}$ In particular, $X$ is quasi-compact and separated.
} 
and $A=\mathcal{D}_{X}$ ), one hence obtains a motivic isomorphism

$$
U\left(\operatorname{perf}_{\mathrm{dg}}\left(T^{*} X^{(1)}\right)\right)_{R} \simeq U\left(\operatorname{perf}_{\mathrm{dg}}\left(\mathcal{D}_{X}\right)\right)_{R}
$$

for every commutative ring $R$ containing $1 / p$.

Corollary 3.8. Let $k, X, R$ be as above, and $E: \operatorname{dgcat}(k) \rightarrow \mathrm{D}$ an additive invariant with values in a $R$-linear category. Under these assumptions, one has an isomorphism $E\left(T^{*} X^{(1)}\right) \simeq E\left(\mathcal{D}_{X}\right)$.

Cubic fourfolds containing a plane. Let $k=\mathbb{C}$ and $X$ a (generic) cubic fourfold, i.e. a smooth complex hypersurface of degree $3 \mathrm{in} \mathbb{P}^{5}$. In the case where $X$ contains a plane, Kuznetsov constructed in [27] a semi-orthogonal decomposition

$$
\operatorname{perf}(X)=\left(\operatorname{perf}\left(B_{S}\right), \mathcal{O}_{X}, \mathcal{O}_{X}(1), \mathcal{O}_{X}(2)\right),
$$

where $S$ is a smooth projective complex $K 3$-surface and $B_{S}$ a sheaf of Azumaya algebras over $S$ of rank 4. By combining Theorem 2.1 (with $X=S$ and $A=B_{S}$ ) with [30, Lem. 5.1], one hence obtains the following motivic decomposition

$$
U\left(\operatorname{perf}_{\mathrm{dg}}(X)\right)_{R} \simeq U\left(\operatorname{perf}_{\mathrm{dg}}(S)\right)_{R} \oplus U(\underline{\mathbb{C}})_{R}^{\oplus 3}
$$

for every commutative ring $R$ containing $1 / 2$.

Corollary 3.9. Let $X, S, R$ be as above, and $E: \operatorname{dgcat}(\mathbb{C}) \rightarrow \mathrm{D}$ an additive invariant with values in a $R$-linear category. Under these assumptions, one has an isomorphism $E(X) \simeq E(S) \oplus E(\mathbb{C})^{\oplus 3}$.

Severi-Brauer varieties. Let $k$ be a field, $A$ a central simple $k$-algebra of degree $\sqrt{\operatorname{dim}(A)}=d$, and $S B(A)$ the associated Severi-Brauer variety. As proved in [4, Prop. 2.8], one has the following motivic decomposition

$$
U\left(\operatorname{perf}_{\mathrm{dg}}(S B(A))\right)_{R} \simeq U(\underline{k})_{R} \oplus U(\underline{A})_{R} \oplus U(\underline{A})_{R}^{\otimes 2} \oplus \cdots \oplus U(\underline{A})_{R}^{\otimes d-1}
$$

for every commutative ring $R$. Consequently, Theorem 2.1 (with $X=\operatorname{Spec}(k)$ ) combined with the fact that $U(\underline{k})_{R}$ is the $\otimes$-unit of $\mathrm{Hmo}_{0}(k)_{R}$, allows us to conclude that whenever $1 / d \in R,(3.10)$ reduces to

$$
U\left(\operatorname{perf}_{\mathrm{dg}}(S B(A))\right)_{R} \simeq \underbrace{U(\underline{k})_{R} \oplus \cdots \oplus U(\underline{k})_{R}}_{d \text {-copies }} .
$$

Corollary 3.11. Let $A, R$ be as above, and $E: \operatorname{dgcat}(k) \rightarrow \mathrm{D}$ an additive invariant with values in a $R$-linear category. Under these assumptions, one has an isomorphism $E(S B(A)) \simeq E(k)^{\oplus d}$.

Clifford algebras. Let $k$ be a field of characteristic $\neq 2, V$ a finite dimensional $k$-vector space of dimension $n$, and $q: V \rightarrow k$ a non-degenerate quadratic form. Recall from $[28, \S \mathrm{V}]$ that out of this data one can construct the Clifford algebra $C(q)$, the even Clifford algebra $C_{0}(q)$, and the signed determinant $\delta(q) \in k^{\times} /\left(k^{\times}\right)^{2}$. The $k$-algebra $C(q)$ has dimension $2^{n}$ and $C_{0}(q)$ dimension $2^{n-1}$. When $n$ is odd we have the following structure results (see [28, §V Thm. 2.4]):

(i) $C_{0}(q)$ is a central simple $k$-algebra;

(ii) When $\delta(q) \notin\left(k^{\times}\right)^{2}, C(q)$ is a central simple algebra over its center $k(\sqrt{\delta(q)})$;

(iii) When $\delta(q) \in\left(k^{\times}\right)^{2}, C(q)$ is a product of two isomorphic central simple algebras over the center $k \times k$. 
Using Theorem 2.1 we then obtain the following motivic decompositions

$$
U\left(\underline{C_{0}(q)}\right)_{R} \simeq U(\underline{k})_{R} \quad U(\underline{C(q)})_{R} \simeq\left\{\begin{array}{lll}
U(k(\sqrt{\delta(q)}))_{R} & \text { when } \delta(q) \notin\left(k^{\times}\right)^{2} \\
U\left(\underline{\underline{k})_{R} \oplus U(\underline{k})_{R}}\right. & \text { when } \delta(q) \in\left(k^{\times}\right)^{2}
\end{array}\right.
$$

for every commutative ring $R$ containing $1 / 2$. When $n$ is even we have the (opposite) structure results (see [28, §V Thm. 2.5]):

(i') $C(q)$ is a central simple $k$-algebra;

(ii') When $\delta(q) \notin\left(k^{\times}\right)^{2}, C_{0}(q)$ is a central simple algebra over its center $k(\sqrt{\delta(q)})$;

(iii') When $\delta(q) \in\left(k^{\times}\right)^{2}, C_{0}(q)$ is a product of two isomorphic central simple algebras over the center $k \times k$.

Using Theorem 2.1 once again we obtain the motivic decompositions

$$
U(\underline{C(q)})_{R} \simeq U(\underline{k})_{R} \quad U\left(\underline{C_{0}(q)}\right)_{R} \simeq\left\{\begin{array}{lll}
U(k(\sqrt{\delta(q)}))_{R} & \text { when } & \delta(q) \notin\left(k^{\times}\right)^{2} \\
U(\underline{k})_{R} \oplus U(\underline{k})_{R} & \text { when } & \delta(q) \in\left(k^{\times}\right)^{2}
\end{array}\right.
$$

for every commutative ring $R$ containing $1 / 2$. Thanks to Corollary 3.1, the above four isomorphisms hold also with $U$ replaced by any additive invariant $E$ with values in a $R$-linear category.

Quadrics. Let $k, q$ be as in the previous subsection (with $n \geq 3$ ), and $Q_{q} \subset \mathbb{P}(V)$ the associated smooth projective quadric of dimension $n-2$. As explained in the proof of [4, Prop. 2.3], one has the following motivic decomposition

$$
U\left(\operatorname{perf}_{\mathrm{dg}}\left(Q_{q}\right)\right)_{R} \simeq U({\underline{C_{0}(q)}}_{R} \oplus \underbrace{U(\underline{k})_{R} \oplus \cdots \oplus U(\underline{k})_{R}}_{(n-2) \text {-copies }}
$$

for every commutative ring $R$. By combing it with Corollary 3.1 and with the four isomorphisms of the previous subsection, we obtain the following result:

Corollary 3.12. Let $k, q$ be as above, $R$ a commutative ring containing $1 / 2$, and $E: \operatorname{dgcat}(k) \rightarrow \mathrm{D}$ an additive invariant with values in a R-linear category. Under these assumptions, one has an isomorphism between $E\left(Q_{q}\right)$ and:

(i) $E(k)^{\oplus n-1}$ when $n$ is odd;

(ii) $E(k)^{\oplus n}$ when $n$ is even and $\delta(q) \in\left(k^{\times}\right)^{2}$;

(iii) $E\left(C_{0}(q)\right) \oplus E(k)^{\oplus n-1}$ when $n$ is even and $\delta(q) \notin\left(k^{\times}\right)^{2}$.

Finite dimensional algebras of finite global dimension. Let $k$ be a field of characteristic $p \geq 0$ and $R$ a commutative ring. We start by describing the behavior of the universal additive invariant with respect to nilpotent extensions.

Theorem 3.13. (Nilinvariance) Let $S$ be a finite dimensional k-algebra of finite global dimension and $I \subset S$ a nilpotent (two-sided) ideal. Assume that:

(i) the k-algebra $S / I$ has finite global dimension;

(ii) the quotient of $S$ by its Jacobson radical $J(S)$ is $k$-separable (e.g. $k$ perfect) or that $1 / p \in R$.

Under the above assumptions, one has an induced isomorphism $U(\underline{S})_{R} \stackrel{\sim}{\rightarrow} U(\underline{S / I})_{R}$.

In the particular case where $I=J(S)$ the above assumption (i) holds automatically since $S / J(S)$ is semi-simple. Hence, modulo assumption (ii), Theorem 3.13 shows us that the noncommutative motives of a finite dimensional algebra of finite global dimension and of its largest semi-simple quotient are isomorphic. 
Corollary 3.14. Let $k, S, I, R$ be as in Theorem 3.13, and $E: \operatorname{dgcat}(k) \rightarrow \mathrm{D}$ an additive invariant with values in an R-linear category. Under these assumptions, one has an isomorphism $E(S) \simeq E(S / I)$. In particular, $E(S) \simeq E(S / J(S))$.

The isomorphisms $K_{n}(S) \simeq K_{n}(S / I), n \geq 0$, are well-known. The case $n=0$ follows from idempotent lifting (see [42, page 70]) and the remaining cases from dévissage (see [42, page 118]). All the remaining isomorphisms provided by Corollary 3.14 are, to the best of the authors knowledge, new in the literature.

Remark 3.15. Theorem 3.13 is false when $S$ is of infinite global dimension. An example is given by the $k$-algebra $S:=k[\epsilon] / \epsilon^{2}$ of dual numbers and by the ideal $I:=$ $\epsilon S$. Since $S$ and $S / \epsilon S \simeq k$ are local $k$-algebras one has the following isomorphisms

$$
K_{1}\left(k[\epsilon] / \epsilon^{2}\right) \simeq\left(k[\epsilon] / \epsilon^{2}\right)^{*}=k^{*}+k \epsilon \quad K_{1}(S / \epsilon S) \simeq K_{1}(k) \simeq k^{*} ;
$$

see [42, page 183]. This implies that the induced map $K_{1}(S) \stackrel{\epsilon=0}{\rightarrow} K_{1}(S / \epsilon S)$ is not an isomorphism and so using Corollary 3.14 one concludes that $U(\underline{S}) \stackrel{\epsilon=0}{\rightarrow} U(S / \epsilon S)$ is not an isomorphism. Note that in the particular case where $k$ is a finite field, the groups (3.16) are not even abstractly isomorphic because they have different cardinality. In this case we hence have $U(\underline{S}) \neq U(S / \epsilon S)$.

Let $V_{1}, \ldots, V_{m}$ be the simple (right) $S$-modules and $D_{1}:=\operatorname{End}_{S}\left(V_{1}\right), \ldots, D_{m}:=$ $\operatorname{End}_{S}\left(V_{m}\right)$ the associated division $k$-algebras. Thanks to the Artin-Wedderburn theorem, the quotient $S / J(S)$ is Morita equivalent to $D_{1} \times \cdots \times D_{m}$. The center $Z_{i}$ of $D_{i}$ is a finite field extension of $k$ and $D_{i}$ is a central simple $Z_{i}$-algebra. Let $r_{i}:=\left[D_{i}: Z_{i}\right]$ and $r:=r_{1} \times \cdots \times r_{m}$. Using Theorem 2.1 (with $X=\operatorname{Spec}\left(Z_{i}\right)$ and $A=D_{i}$ ), one then obtains the following isomorphism

$$
U(\underline{S / J(S)})_{R} \simeq U\left(\underline{Z_{i}}\right)_{R} \oplus \cdots \oplus U\left(\underline{Z_{m}}\right)_{R}
$$

for every commutative ring $R$ containing $1 / r$ or $1 /(r p)$ depending on whether we assume that $S / J(S)$ is $k$-separable or not. The combination of (3.17) with the above Corollary 3.14 gives rise to the following result:

Corollary 3.18. Let $k, S, Z_{i}, R$ be as above, and $E: \operatorname{dgcat}(k) \rightarrow \mathrm{D}$ an additive invariant with values in a $R$-linear category. Under the assumptions of Theorem 3.13, one has an isomorphism $E(S) \simeq E\left(Z_{1}\right) \oplus \cdots \oplus E\left(Z_{m}\right)$.

Intuitively speaking, Corollary 3.18 shows us that all additive invariants of finite dimensional $k$-algebras of finite global dimension can be computed using solely finite field extensions of $k$.

Remark 3.19. When $k$ is algebraically closed, we have $D_{1}=\cdots=D_{m}=k$ and consequently $Z_{1}=\cdots=Z_{m}=k$. Corollary 3.18 reduces then (for every commutative ring $R$ ) to an isomorphism $E(S) \simeq E(k)^{\oplus m}$. This isomorphism was also obtained by Keller in [20, §2.3] using different arguments.

Notations. Throughout the article we will reserve the letter $k$ for a base commutative ring, the letter $R$ for a commutative ring of coefficients, the letters $A, B, C$ for sheaves of Azumaya algebras over schemes, the letters $\mathcal{A}, \mathcal{B}$ for dg categories, the letters $S, T$ for $(\mathrm{dg}) k$-algebras, and finally the letters $X, Y$ for $k$-schemes. All schemes will be assumed to be quasi-compact and quasi-separated. Given a small category $\mathcal{C}$, we will write Iso $\mathcal{C}$ for its set of isomorphism classes of objects. 
Acknowledgments: The authors are grateful to the Mathematical Science Research Institute (MSRI) in Berkeley, California, for its hospitality and excellent working conditions. They would also like to thank Ben Antieau for pointing out Gabber's work [14] as well for indicating an alternative proof to Theorem 2.3.

\section{BACKGROUND ON DG CATEGORIES}

Let $\mathcal{C}(k)$ be the category of cochain complexes of $k$-modules; we use cohomological notation. A differential graded $(=d g)$ category $\mathcal{A}$ is a category enriched over $\mathcal{C}(k)$ (morphisms sets $\mathcal{A}(x, y)$ are complexes) in such a way that composition fulfills the Leibniz rule $d(f \circ g)=d(f) \circ g+(-1)^{\operatorname{deg}(f)} f \circ d(g)$. A dg functor $F: \mathcal{A} \rightarrow \mathcal{B}$ is a functor enriched over $\mathcal{C}(k)$; consult Keller's ICM survey [17]. In what follows we will write $\operatorname{dgcat}(k)$ for the category of (small) dg categories and dg functors.

Modules. Let $\mathcal{A}$ be a dg category. The category $\mathrm{H}^{0}(\mathcal{A})$ has the same objects as $\mathcal{A}$ and morphisms given by $\mathrm{H}^{0}(\mathcal{A})(x, y):=H^{0}(\mathcal{A}(x, y))$, where $H^{0}$ denotes degree zero cohomology. The opposite $\mathrm{dg}$ category $\mathcal{A}^{\mathrm{op}}$ has the same objects as $\mathcal{A}$ and complexes of morphisms given by $\mathcal{A}^{\mathrm{op}}(x, y):=\mathcal{A}(y, x)$. A right $\mathcal{A}$-module is a $\mathrm{dg}$ functor $\mathcal{A}^{\mathrm{op}} \rightarrow \mathcal{C}_{\mathrm{dg}}(k)$ with values in the dg category $\mathcal{C}_{\mathrm{dg}}(k)$ of cochain complexes of $k$-modules. Let us denote by $\mathcal{C}(\mathcal{A})$ the category of right $\mathcal{A}$-modules. Recall from $[17$, $\S 3.2]$ that the derived category $\mathcal{D}(\mathcal{A})$ of $\mathcal{A}$ is the localization of $\mathcal{C}(\mathcal{A})$ with respect to the class of objectwise quasi-isomorphisms. Its full subcategory of compact objects will be denoted by $\mathcal{D}_{c}(\mathcal{A})$.

Morita equivalences. A dg functor $F: \mathcal{A} \rightarrow \mathcal{B}$ is called a Morita equivalence if the induced restriction of scalars $\mathcal{D}(\mathcal{B}) \stackrel{\sim}{\rightarrow} \mathcal{D}(\mathcal{A})$ is an equivalence of (triangulated) categories; see [17, §4.6]. As proved in [37, Thm. 5.3], dgcat $(k)$ admits a Quillen model structure whose weak equivalences are precisely the Morita equivalences. Let us denote by $\mathrm{Hmo}(k)$ the homotopy category hence obtained.

Tensor product. The tensor product $\mathcal{A} \otimes \mathcal{B}$ of two dg categories $\mathcal{A}$ and $\mathcal{B}$ is defined by the cartesian product of the sets of objects of $\mathcal{A}$ and $\mathcal{B}$ and by the complexes of morphisms $(\mathcal{A} \otimes \mathcal{B})((x, z),(y, w)):=\mathcal{A}(x, y) \otimes \mathcal{B}(z, w)$. As explained in [17, §2.3], this gives rise to a symmetric monoidal structure on $\operatorname{dgcat}(k)$ with $\otimes$-unit the $\mathrm{dg}$ category $\underline{k}$. After deriving it $-\otimes^{\mathbb{L}}-$, this symmetric monoidal structure descends to $\mathrm{Hmo}(k)$; consult $[17, \S 4.3]$ for details.

Bimodules. Let $\mathcal{A}, \mathcal{B} \in \operatorname{dgcat}(k)$. $\mathrm{A} \mathcal{A}$ - $\mathcal{B}$-bimodule $\mathrm{B}$ is a right $\left(\mathcal{A}^{\mathrm{op}} \otimes \mathcal{B}\right)$-module, i.e. a dg functor $\mathrm{B}: \mathcal{A} \otimes \mathcal{B}^{\mathrm{op}} \rightarrow \mathcal{C}_{\mathrm{dg}}(k)$. Standard examples are the $\mathcal{A}$ - $\mathcal{A}$-bimodule

$$
\mathcal{A} \otimes \mathcal{A}^{\mathrm{op}} \rightarrow \mathcal{C}_{\mathrm{dg}}(k) \quad(x, y) \mapsto \mathcal{A}(y, x)
$$

as well as the $\mathcal{A}$ - $\mathcal{B}$-bimodule

$$
{ }_{F} \mathrm{Bi}: \mathcal{A} \otimes \mathcal{B}^{\mathrm{op}} \rightarrow \mathcal{C}_{\mathrm{dg}}(k) \quad(x, z) \mapsto \mathcal{B}(z, F(x))
$$

associated to a dg functor $F: \mathcal{A} \rightarrow \mathcal{B}$.

Smoothness and properness. Recall from Kontsevich [21, 22, 23, 24] that a $\mathrm{dg}$ category $\mathcal{A}$ is called smooth if the $\mathcal{A}$ - $\mathcal{A}$-bimodule (4.1) belongs to $\mathcal{D}_{c}\left(\mathcal{A}^{\mathrm{op}} \otimes^{\mathbb{L}} \mathcal{A}\right)$ and proper if for each ordered pair of objects $(x, y)$ we have $\sum_{i} \operatorname{rank} H^{i} \mathcal{A}(x, y)<\infty$. 


\section{BACKGROUND ON NONCOMMUTATIVE MOTIVES}

In this section we recall from [37] the construction of the category of noncommutative motives; consult also the survey article [34]. Let $\mathcal{A}, \mathcal{B} \in \operatorname{dgcat}(k)$. As proved in [37, Cor. 5.10], one has a bijection

$$
\operatorname{Hom}_{\operatorname{Hmo}(k)}(\mathcal{A}, \mathcal{B}) \simeq \operatorname{Iso} \operatorname{rep}(\mathcal{A}, \mathcal{B})
$$

where $\operatorname{rep}(\mathcal{A}, \mathcal{B})$ denotes the full triangulated subcategory of $\mathcal{D}\left(\mathcal{A}^{\mathrm{op}} \otimes \otimes^{\mathbb{L}} \mathcal{B}\right)$ consisting of those $\mathcal{A}$ - $\mathcal{B}$-bimodules $\mathrm{B}$ such that for every $x \in \mathcal{A}$ the right $\mathcal{B}$-module $\mathrm{B}(x,-)$ belongs to $\mathcal{D}_{c}(\mathcal{B})$. Under (5.1), the composition law in $\mathrm{Hmo}(k)$ corresponds to the (derived) tensor product of bimodules and the identity of an object $\mathcal{A}$ is given by the isomorphism class of the $\mathcal{A}$ - $\mathcal{A}$-bimodule (4.1). Since the $\mathcal{A}$ - $\mathcal{B}$-bimodules (4.2) belong to $\operatorname{rep}(\mathcal{A}, \mathcal{B})$, we hence obtain a well-defined symmetric monoidal functor

$$
\operatorname{dgcat}(k) \longrightarrow \operatorname{Hmo}(k) \quad F \mapsto{ }_{F} \mathrm{Bi} .
$$

The additivization of $\mathrm{Hmo}(k)$ is the additive category $\mathrm{Hmo}_{0}(k)$ with the same objects as $\mathrm{Hmo}(k)$ and abelian groups of morphisms given by

$$
\operatorname{Hom}_{\mathrm{Hmo}_{0}(k)}(\mathcal{A}, \mathcal{B}):=K_{0} \operatorname{rep}(\mathcal{A}, \mathcal{B}),
$$

where $K_{0} \operatorname{rep}(\mathcal{A}, \mathcal{B})$ is the Grothendieck group of the triangulated category $\operatorname{rep}(\mathcal{A}, \mathcal{B})$. The composition law is induced by the (derived) tensor product of bimodules. Note that we have also a canonical functor

$$
\mathrm{Hmo}(k) \longrightarrow \mathrm{Hmo}_{0}(k) \quad \mathrm{B} \mapsto[\mathrm{B}] .
$$

Finally, given a commutative ring of coefficients $R$, the $R$-linearization of $\mathrm{Hmo}_{0}(k)$ is the $R$-linear additive category $\mathrm{Hmo}_{0}(k)_{R}$ obtained by tensoring each abelian group of morphisms of $\mathrm{Hmo}_{0}(k)$ with $R$. This gives rise to a functor

$$
\mathrm{Hmo}_{0}(k) \longrightarrow \mathrm{Hmo}_{0}(k)_{R} \quad[\mathrm{~B}] \mapsto[\mathrm{B}] \otimes_{\mathbb{Z}} R .
$$

As proved in [37], the symmetric monoidal structure on $\mathrm{Hmo}(k)$ descends first to a bilinear symmetric monoidal structure on $\mathrm{Hmo}_{0}(k)$ and then to a $R$-linear bilinear symmetric monoidal structure on $\mathrm{Hmo}_{0}(k)_{R}$, making (5.3)-(5.4) into symmetric monoidal functors. The universal additive invariant with $R$-coefficients (1.1) is then defined by the following composition

$$
U(-)_{R}: \operatorname{dgcat}(k) \stackrel{(5.2)}{\longrightarrow} \mathrm{Hmo}(k) \stackrel{(5.3)}{\longrightarrow} \mathrm{Hmo}_{0}(k) \stackrel{(5.4)}{\longrightarrow} \mathrm{Hmo}_{0}(k)_{R} .
$$

Finally, given $\operatorname{dg}$ categories $\mathcal{A}, \mathcal{B} \in \operatorname{dgcat}(k)$ with $\mathcal{A}$ smooth and proper, the triangulated category $\operatorname{rep}(\mathcal{A}, \mathcal{B}) \subset \mathcal{D}\left(\mathcal{A}^{\mathrm{op}} \otimes^{\mathbb{L}} \mathcal{B}\right)$ identifies with $\mathcal{D}_{c}\left(\mathcal{A}^{\mathrm{op}} \otimes^{\mathbb{L}} \mathcal{B}\right)$; see $[11$, $\S 5]$. As a consequence, we obtain the following isomorphism

$$
\operatorname{Hom}_{\mathrm{Hmo}_{0}(k)_{R}}\left(U(\mathcal{A})_{R}, U(\mathcal{B})_{R}\right) \simeq K_{0}\left(\mathcal{A} \otimes^{\mathbb{L}} \mathcal{B}\right)_{R} .
$$

\section{Perfect complexes}

Let $k$ be a base commutative ring, $X$ a quasi-compact quasi-separated $k$-scheme, and $A$ a sheaf of $\mathcal{O}_{X}$-algebras. We introduce some notations and concepts that are standard in the particular case where $A=\mathcal{O}_{X}$ (consult $[9, \S 3][17, \S 4.4]$ and the references therein) and whose generalization to an arbitrary sheaf $A$ is immediate.

Let $\operatorname{Mod}(A)$ be the Grothendieck category of sheaves of (right) $A$-modules, $\mathrm{Q} \operatorname{coh}(A)$ the full subcategory of quasi-coherent $A$-modules, $\mathcal{D}(A):=D(\operatorname{Mod}(A))$ 
the derived category of $A$, and $\mathcal{D}_{\mathrm{Qcoh}}(A) \subset \mathcal{D}(A)$ the full triangulated subcategory of those complexes of $A$-modules with quasi-coherent cohomology. When $X$ is separated we have $\mathcal{D}_{\mathrm{Qcoh}}(A) \simeq D(\mathrm{Q} \operatorname{coh}(A))$.

Definition 6.1. A complex of $A$-modules $\mathcal{F} \in \mathcal{D}(A)$ is called perfect if there exists a covering $X=\bigcup_{i} V_{i}$ of $X$ by affine open subschemes $V_{i} \subset X$ such that for every $i$ the restriction $\mathcal{F}_{\mid V_{i}}$ of $\mathcal{F}$ to $V_{i}$ is quasi-isomorphic to a bounded complex of finitely generated projective $A_{\mid V_{i}}$-modules. Let us denote by $\operatorname{perf}(A)$ the triangulated category of perfect complexes. Note that by construction we have the inclusions $\operatorname{perf}(A) \subset \mathcal{D}_{\mathrm{Qcoh}}(A) \subset \mathcal{D}(A)$.

Let $\mathcal{E}$ be an abelian (or exact) category. As explained in [17, §4.4], the derived dg category $\mathcal{D}_{\mathrm{dg}}(\mathcal{E})$ of $\mathcal{E}$ is defined as the $\operatorname{dg}$ quotient $\mathcal{C}_{\mathrm{dg}}(\mathcal{E}) / \mathcal{A} c_{\mathrm{dg}}(\mathcal{E})$ of the dg category of complexes over $\mathcal{E}$ by its full dg subcategory of acyclic complexes. Note that every exact functor $\mathcal{E} \rightarrow \mathcal{E}^{\prime}$ (or more generally every dg functor $\mathcal{C}_{\mathrm{dg}}(\mathcal{E}) \rightarrow \mathcal{C}_{\mathrm{dg}}\left(\mathcal{E}^{\prime}\right.$ ) which restricts to $\mathcal{A} c_{\mathrm{dg}}(\mathcal{E}) \rightarrow \mathcal{A} c_{\mathrm{dg}}\left(\mathcal{E}^{\prime}\right)$ ) gives rise to a dg functor $\mathcal{D}_{\mathrm{dg}}(\mathcal{E}) \rightarrow \mathcal{D}_{\mathrm{dg}}\left(\mathcal{E}^{\prime}\right)$.

Notation 6.2. Let us write $\mathcal{D}_{\mathrm{dg}}(A)$ for the dg category $\mathcal{D}_{\mathrm{dg}}(\mathcal{E})$ with $\mathcal{E}:=\operatorname{Mod}(A)$, $\mathcal{D}_{\text {Qcoh,dg }}(A)$ for the full dg category of those complexes of $A$-modules with quasicoherent cohomology, and $\operatorname{perf}_{\mathrm{dg}}(A)$ for the full dg subcategory of perfect complexes. By construction, we have inclusions perf $\operatorname{dg}_{\mathrm{dg}}(A) \subset \mathcal{D}_{\mathrm{Qcoh}, \mathrm{dg}}(A) \subset \mathcal{D}_{\mathrm{dg}}(A)$ of $\mathrm{dg}$ categories and canonical equivalences

$$
\mathrm{H}^{0}\left(\operatorname{perf}_{\mathrm{dg}}(A)\right) \simeq \operatorname{perf}(A) \quad \mathrm{H}^{0}\left(\mathcal{D}_{\mathrm{Qcoh}, \mathrm{dg}}(A)\right) \simeq \mathcal{D}_{\mathrm{Qcoh}}(A) \quad \mathrm{H}^{0}\left(\mathcal{D}_{\mathrm{dg}}(A)\right) \simeq \mathcal{D}(A) .
$$

When $A=\mathcal{O}_{X}$ we will write in what follows $X$ instead of $\mathcal{O}_{X}$.

Note that we have a well-defined forgetful functor $\mathcal{D}(A) \rightarrow \mathcal{D}(X)$ which restricts to $\operatorname{perf}(A) \rightarrow \operatorname{perf}(X)$ when $A$ is perfect as a complex of $\mathcal{O}_{X}$-modules. Since the forgetful functor $\operatorname{Mod}(A) \rightarrow \operatorname{Mod}(X)$ is exact, one has similar forgetful dg functors $\mathcal{D}_{\mathrm{dg}}(A) \rightarrow \mathcal{D}_{\mathrm{dg}}(X)$ and $\operatorname{perf}_{\mathrm{dg}}(A) \rightarrow \operatorname{perf}_{\mathrm{dg}}(X)$. The following (well-known) fact will be used in the sequel.

Lemma 6.3. Let $X$ and $A$ be as above, with $A$ a sheaf of Azumaya algebras over $X$. Under these assumptions, the following square is cartesian

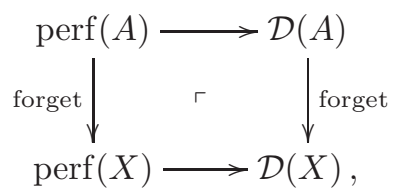

i.e. a complex $\mathcal{F} \in \mathcal{D}(A)$ belongs to $\operatorname{perf}(A)$ if an only if it belongs to $\operatorname{perf}(X)$.

Proof. Thanks to the above Definition 6.1, it suffices to prove the affine case where $X=\operatorname{Spec}(S)$ and $A$ is an Azumaya algebra over $S$. Recall from [26, III §5] that:

(i) $A$ is finitely generated and projective as a right $S$-module;

(ii) $A$ is separable, i.e. $A$ is projective as a $A$-A-bimodule.

If by hypothesis $\mathcal{F}$ belongs to $\operatorname{perf}(A)$, then condition (i) allows us to conclude that $\mathcal{F}$ also belongs to $\operatorname{perf}(X)$. In order to prove the converse implication, consider the base-change functor $-\otimes_{S} A: \mathcal{D}(S) \rightarrow \mathcal{D}(A)$. By construction, it preserves perfect complexes. Hence, if by hypothesis $\mathcal{F}$ belongs to $\operatorname{perf}(X), \mathcal{F} \otimes_{S} A$ belongs to $\operatorname{perf}(A)$. Now, consider the following short exact sequence of $A$ - $A$-bimodules

$$
0 \longrightarrow \operatorname{Ker}(m) \longrightarrow A \otimes_{S} A \stackrel{m}{\longrightarrow} A \longrightarrow 0,
$$


where $m$ stands for the multiplication of $A$. Thanks to the above condition (ii), (6.4) splits and hence $A$ becomes a direct summand of the $A$ - $A$-bimodule $A \otimes_{S} A$. Using the canonical isomorphism $\mathcal{F} \otimes_{S} A \simeq \mathcal{F} \otimes_{A}\left(A \otimes_{S} A\right)$ one concludes that $\mathcal{F}$ is a direct summand of $\mathcal{F} \otimes_{S} A$. Since $\mathcal{F} \otimes_{S} A$ belongs to $\operatorname{perf}(A)$ and this category is idempotent complete, $\mathcal{F}$ also belongs to $\operatorname{perf}(A)$. This completes the proof.

Every sheaf $A$ of $\mathcal{O}_{X}$-algebras gives rise to the following dg functor

$$
-\otimes_{\mathcal{O}_{X}}^{\mathbb{L}} A: \mathcal{C}_{\mathrm{dg}}(\operatorname{Mod}(X)) \longrightarrow \mathcal{C}_{\mathrm{dg}}(\operatorname{Mod}(A)) \quad \mathcal{F} \mapsto \mathcal{F}_{\text {flat }} \otimes_{\mathcal{O}_{X}} A,
$$

where $\mathcal{F}_{\text {flat }}$ denotes a (functorial) $\mathcal{O}_{X}$-flat resolution of $\mathcal{F}$. Note that when $A$ is $\mathcal{O}_{X}$-flat (e.g. $A$ locally free of finite rank over $\mathcal{O}_{X}$ ), (6.5) identifies with $-\otimes_{\mathcal{O}_{X}} A$. Since (6.5) preserves acyclic and perfect complexes, it induces a dg functor

$$
-\otimes_{\mathcal{O}_{X}}^{\mathbb{L}} A: \operatorname{perf}_{\mathrm{dg}}(X) \longrightarrow \operatorname{perf}_{\mathrm{dg}}(A) .
$$

\section{Proof of Theorem 2.3}

Since $X$ is quasi-compact and quasi-separated, the proof can be reduced to the affine case and to a Mayer-Vietoris argument; see [9, Prop. 3.3.1].

Lemma 7.1. (Thomason-Trobaugh $[39, \S 8]$ ) Let $X$ be a quasi-compact quasi-separated scheme, and $U_{1}, U_{2}$ two Zariski open subschemes. Assume that $X=U_{1} \cup U_{2}$ and write $U_{12}:=U_{1} \cap U_{2}$. Under these assumptions, one has the exact sequence.

$$
K_{1}\left(U_{1}\right) \oplus K_{1}\left(U_{2}\right) \rightarrow K_{1}\left(U_{12}\right) \stackrel{\partial}{\rightarrow} K_{0}(X) \stackrel{ \pm}{\rightarrow} K_{0}\left(U_{1}\right) \oplus K_{0}\left(U_{2}\right) \rightarrow K_{0}\left(U_{12}\right) .
$$

Proof. Let us write $\iota_{1}: U_{1} \hookrightarrow X$ and $\iota_{2}: U_{2} \hookrightarrow X$ for the two open inclusions. Consider the following commutative diagram in $\operatorname{Hmo}(k)$

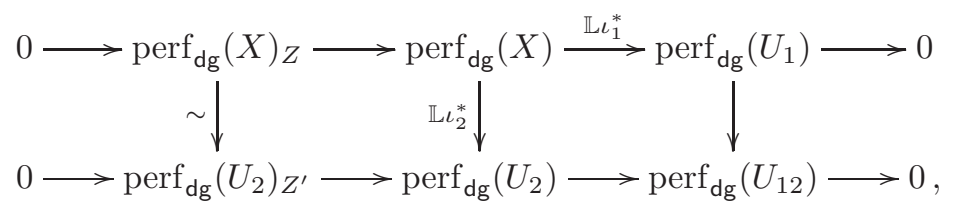

where $Z$ (resp. $Z^{\prime}$ ) is the closed set $X-U_{1}$ (resp. $U_{2}-U_{12}$ ) and perf ${ }_{\mathrm{dg}}(X)_{Z}$ (resp. perf $\left._{\mathrm{dg}}\left(U_{2}\right)_{Z^{\prime}}\right)$ the dg category of those perfect complexes of $\mathcal{O}_{X}$-modules (resp. of $\mathcal{O}_{U_{2}}$-modules) that are supported on $Z$ (resp. on $Z^{\prime}$ ). Recall from $[17, \S 4.6]$ the notion of a short exact sequence of $\mathrm{dg}$ categories. Roughly speaking, it consists of a sequence of dg categories $\mathcal{A} \rightarrow \mathcal{B} \rightarrow \mathcal{C}$ for which $\mathcal{D}(\mathcal{A}) \rightarrow \mathcal{D}(\mathcal{B}) \rightarrow \mathcal{D}(\mathcal{C})$ is exact in the sense of Verdier. As explained in [39, §5], both rows in (7.3) are short exact sequences of $\mathrm{dg}$ categories; see also [17, §4.6]. Furthermore, as proved in [39, Thm. 2.6.3], the induced dg functor $\operatorname{perf}_{\mathrm{dg}}(X)_{Z} \stackrel{\sim}{\rightarrow} \operatorname{perf}_{\mathrm{dg}}\left(U_{2}\right)_{Z^{\prime}}$ is a Morita equivalence and hence an isomorphism in $\mathrm{Hmo}(k)$.

Nonconnective algebraic $K$-theory gives rise to a functor $\mathbb{K}: \mathrm{Hmo}(k) \rightarrow \mathrm{Ho}(\mathrm{Spt})$ with values in the homotopy category of spectra. Among other properties, it sends short exact sequences of dg categories to distinguished triangles of spectra; see [33][36, Thm. 10.9]. Hence, by applying it to (7.3) we obtain the following morphism 
between distinguished triangles

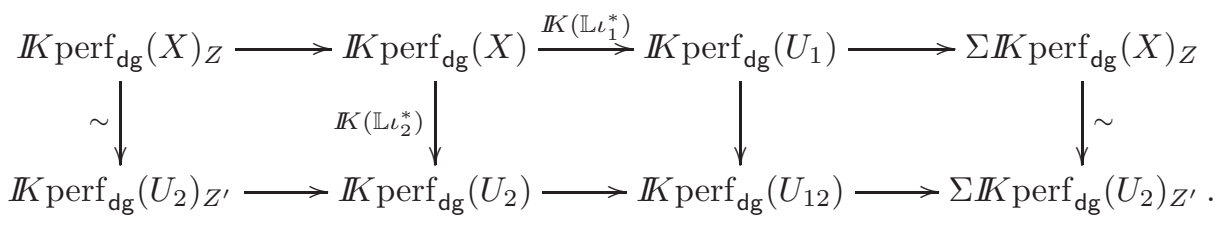

Since the outer left and right vertical maps are isomorphisms we hence obtain a Mayer-Vietoris long exact sequence

$$
\cdots \rightarrow K_{n+1}\left(U_{1}\right) \oplus K_{n+1}\left(U_{2}\right) \rightarrow K_{n+1}\left(U_{12}\right) \stackrel{\partial}{\rightarrow} K_{n}(X) \stackrel{\text { 与 }}{\rightarrow} K_{n}\left(U_{1}\right) \oplus K_{n}\left(U_{2}\right) \rightarrow \cdots,
$$

where the boundary maps $\partial$ 's are obtained from the composition

$$
\mathbb{K} \operatorname{perf}_{\mathrm{dg}}\left(U_{12}\right) \longrightarrow \Sigma \mathbb{K} \operatorname{perf}_{\mathrm{dg}}\left(U_{2}\right)_{Z^{\prime}} \stackrel{\sim}{\longrightarrow} \Sigma \mathbb{K}_{\operatorname{perf}} \operatorname{dg}_{\mathrm{dg}}(X)_{Z}
$$

The exact sequence (7.2) is a chunk of the above one and so the proof is finished.

Recall now from [42, page 323] that the pairing

$$
-\otimes_{\mathcal{O}_{X}}^{\mathbb{L}}-: \operatorname{perf}(X) \times \operatorname{perf}(X) \longrightarrow \operatorname{perf}(X)
$$

endows $K_{*}(X)$ with a graded-commutative ring structure.

Lemma 7.5. Let $X, U_{1}, U_{2}, U_{12}$ be as in the above Lemma 7.1. Given an element $\alpha$ in $K_{0}(X)$, we have the following commutative diagram

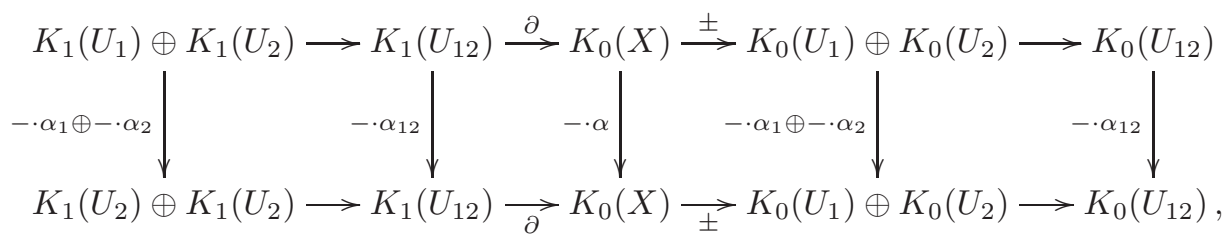

where $\alpha_{1}, \alpha_{2}$ and $\alpha_{12}$ denote the images of $\alpha$ in $K_{0}\left(U_{1}\right), K_{0}\left(U_{2}\right)$ and $K_{0}\left(U_{12}\right)$, respectively.

Proof. Recall first from Thomason $[38, \S 1.6]$ that every element $\alpha$ in $K_{0}(X)$ is of the form $\alpha=[\mathcal{F}]$ for some perfect complex $\mathcal{F}$. Note that we have the following commutative cube in the homotopy category $\mathrm{Hmo}(k)$

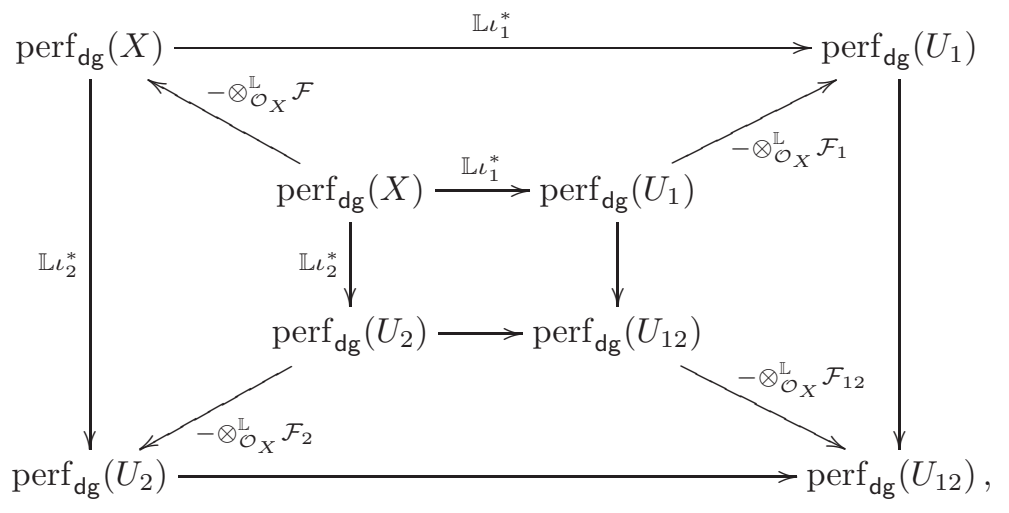


where $\mathcal{F}_{1}, \mathcal{F}_{2}$ and $\mathcal{F}_{12}$ denote the restriction of $\mathcal{F}$ to $U_{1}, U_{2}$ and $U_{12}$, respectively. Following the proof of Lemma 7.1, one observes that the commutative cube (7.6) gives rise to a morphism between Mayer-Vietoris long exact sequences

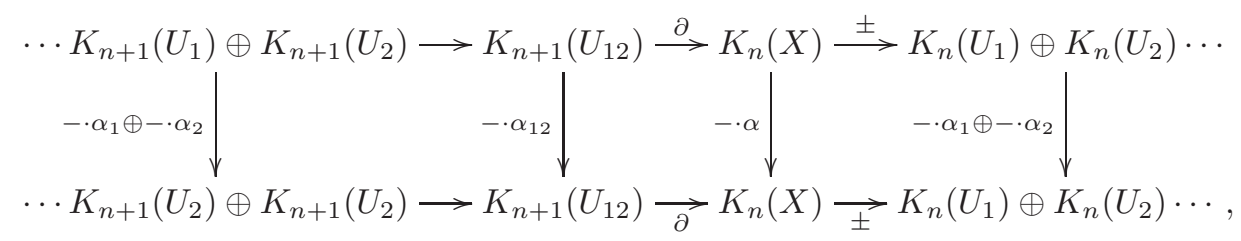

where the commutativity of the middle square follows from composition (7.4). The diagram of Lemma 7.5 is a chunk of this one and so the proof is finished.

Lemma 7.7. Let $X, U_{1}, U_{2}, U_{12}$ and $\alpha, \alpha_{1}, \alpha_{2}, \alpha_{12}$ be as in Lemma 7.5. Whenever $\alpha_{1}, \alpha_{2}$ and $\alpha_{12}$ are nilpotent, $\alpha$ is also nilpotent.

Proof. Since by hypothesis $\alpha_{1}, \alpha_{2}$ and $\alpha_{12}$ are nilpotent, there exists an integer $N \gg 0$ for which the homomorphisms

$$
K_{0}\left(U_{1}\right) \stackrel{-\cdot \alpha_{1}^{N}}{\longrightarrow} K_{0}\left(U_{1}\right) \quad K_{0}\left(U_{2}\right) \stackrel{-\cdot \alpha_{2}^{N}}{\longrightarrow} K_{0}\left(U_{2}\right) \quad K_{1}\left(U_{12}\right) \stackrel{-\cdot \alpha_{2}^{N}}{\longrightarrow} K_{1}\left(U_{12}\right)
$$

are all trivial. Consequently, using the above Lemma 7.5 (with $\alpha$ replaced by $\alpha^{N}$ ), one obtains the following commutative diagram

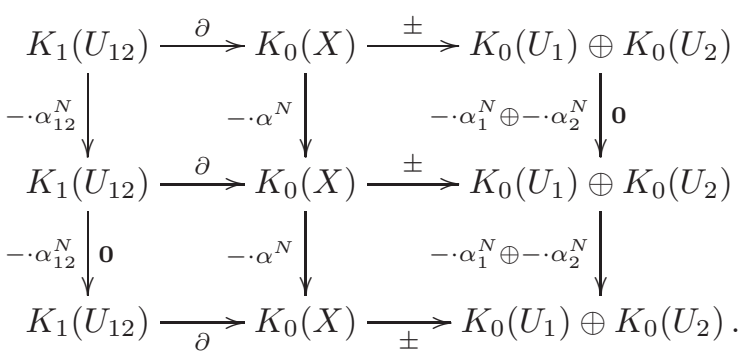

A simple diagram chasing argument then shows that the composition of the middle vertical arrows is zero. I.e. $-\cdot \alpha^{2 N}=0$. This implies that $\alpha^{2 N}=0$ and hence that $\alpha$ is nilpotent.

We now have all the ingredients needed for the proof of Theorem 2.3. Let $\alpha$ be an element in $I_{X} \subset K_{0}(X)$. One needs to show that $\alpha$ is nilpotent. This will be done in three steps.

Step 1. We claim that $X=\bigcup_{i=1}^{n} V_{i}$, where $V_{i} \subset X$ is an affine open subscheme such that the image of $\alpha$ in $K_{0}\left(V_{i}\right)$ is zero. Let $x \in X$ and $V_{x}$ an affine open neighborhood of $x$. The image $\alpha_{\mid V_{x}}$ of $\alpha$ in $K_{0}\left(V_{x}\right)$ can be written as $[P]-[Q]$ with $P$ and $Q$ two vector bundles of the same rank. By shrinking $V_{x}$ we can assume that $P$ and $Q$ are free of the same rank and hence isomorphic. As a consequence, $\alpha_{\mid V_{x}}=0$. Finally, using quasi-compactness, we may take a finite subcover $\left\{V_{i}\right\}_{i=1}^{n}$ of $\left\{V_{x}\right\}_{x \in X}$ which yields the above claim. 
Step 2. Assume that $X$ is a quasi-compact separated scheme. We prove Theorem 2.3 using induction on the number of affine open subschemes in a covering trivializing $\alpha$ as in Step 1. The case $n=1$ is clear. Let us then assume that $n>1$ and write $U_{1}:=\cup_{i=1}^{n-1} V_{i}, U_{2}:=V_{n}$ and $U_{12}:=U_{1} \cap U_{2}$. Since by hypothesis $X$ is separated, $V_{i} \cap V_{j}$ is affine for all $i, j$ and so $U_{1}$ and $U_{12}$ are covered by $n-1$ affine open subschemes on which the restriction of $\alpha$ is trivial. By our induction hypothesis, $\alpha_{1}, \alpha_{2}, \alpha_{12}$ are nilpotent. Hence, using the above Lemma 7.7, we conclude that $\alpha$ is also nilpotent.

Step 3. Assume that $X$ is a quasi-compact quasi-separated scheme. Let $U_{1}, U_{2}$ and $U_{12}$ be as in Step 2. Note that $U_{1}$ is covered by $n-1$ affine open subschemes on which $\alpha$ is trivial and that $U_{2}$ and $U_{12}$ are separated (since $U_{2}$ is affine and $\left.U_{12} \subset U_{2}\right)$. Therefore, using induction and Step 2, we can again assume that $\alpha_{1}$, $\alpha_{2}, \alpha_{12}$ are nilpotent. We finish the proof by invoking Lemma 7.7 once again.

Proof of Corollary 2.4. Every ring homomorphism $R \rightarrow R^{\prime}$ gives rise to a welldefined ring homomorphism $K_{0}(X)_{R} \rightarrow K_{0}(X)_{R^{\prime}}$. Hence, since $\mathbb{Z}[1 / r]$ is initial among the rings containing $1 / r$, it suffices to prove the particular case $R:=\mathbb{Z}[1 / r]$. Note that since $\mathbb{Z}[1 / r]$ is torsion-free we have the following short exact sequence

$$
0 \rightarrow I_{X} \otimes \mathbb{Z}[1 / r] \subset K_{0}(X)_{\mathbb{Z}[1 / r]} \stackrel{\text { rank }}{\rightarrow} \mathbb{Z}^{m} \otimes \mathbb{Z}[1 / r] \rightarrow 0 .
$$

Moreover, thanks to Theorem 2.3, every element in $I_{X} \otimes \mathbb{Z}[1 / r]$ is nilpotent. The rank homomorphism is surjective and so there exists an element $\beta \in K_{0}(X)_{\mathbb{Z}[1 / r]}$ of rank $\left(1 / r_{1}, \ldots, 1 / r_{m}\right)$. Therefore, $\alpha \cdot \beta$ is of $\operatorname{rank}(1, \ldots, 1)$ and consequently $\left(\left[\mathcal{O}_{X}\right]-\alpha \cdot \beta\right) \in I_{X} \otimes \mathbb{Z}[1 / r]$. There exists then an integer $N \gg 0$ such that $\left(\left[\mathcal{O}_{X}\right]-\alpha \cdot \beta\right)^{N+1}=0$. This implies that the following element

$$
\left[\mathcal{O}_{X}\right]+\left(\left[\mathcal{O}_{X}\right]-\alpha \cdot \beta\right)+\left(\left[\mathcal{O}_{X}\right]-\alpha \cdot \beta\right)^{2}+\cdots+\left(\left[\mathcal{O}_{X}\right]-\alpha \cdot \beta\right)^{N} \in K_{0}(X)_{\mathbb{Z}[1 / r]}
$$

is the inverse of $\alpha \cdot \beta$ and hence that $\alpha$ is invertible in $K_{0}(X)_{\mathbb{Z}[1 / r]}$.

\section{Proof of Theorem 2.1}

The proof is divided into two steps. First, we introduce an auxiliary $\mathbb{Z}[1 / r]$-linear category $\mathrm{Az}_{0}(X)_{1 / r}$ of sheaves of Azumaya algebras over $X$ and prove the analogue of Theorem 2.1 therein; see Proposition 8.3. Then, we construct a $\mathbb{Z}[1 / r]$-linear functor from $\mathrm{Az}_{0}(X)_{1 / r}$ to the category $\mathrm{Hmo}_{0}(k)_{\mathbb{Z}[1 / r]}$ of noncommutative motives.

Note that every ring homomorphism $R \rightarrow R^{\prime}$ gives rise to a well-defined additive functor $\mathrm{Hmo}_{0}(k)_{R} \rightarrow \mathrm{Hmo}_{0}(k)_{R^{\prime}}$. Hence, since $\mathbb{Z}[1 / r]$ is initial among the rings containing $1 / r$, it suffices to prove the case $R=\mathbb{Z}[1 / r]$.

Auxiliary category $\mathrm{Az}_{0}(X)$. Given two sheaves $A$ and $B$ of Azumaya algebras over $X$, let $\operatorname{rep}(A, B)$ be the full triangulated subcategory of $\mathcal{D}\left(A^{\mathrm{op}} \otimes_{\mathcal{O}_{X}} B\right)$ consisting of those $A$ - $B$-bimodules ${ }_{A} \mathrm{~B}_{B}$ such that $\mathrm{B}_{B} \in \operatorname{perf}(B)$.

Lemma 8.1. The categories $\operatorname{rep}(A, B)$ and $\operatorname{perf}\left(A^{\mathrm{op}} \otimes_{\mathcal{O}_{X}} B\right)$ are the same.

Proof. We start with the inclusion $\operatorname{rep}(A, B) \subseteq \operatorname{perf}\left(A^{\text {op }} \otimes_{\mathcal{O}_{X}} B\right)$. Let ${ }_{A} \mathrm{~B}_{B}$ be an object of $\operatorname{rep}(A, B) \subset \mathcal{D}\left(A^{\mathrm{op}} \otimes_{\mathcal{O}_{X}} B\right)$. By definition, $\mathrm{B}_{B} \in \operatorname{perf}(B)$. Hence, Lemma 6.3 (with $A=B$ ) shows us that $B \in \operatorname{perf}(X)$. Using again Lemma 6.3 (with $\left.A=A^{\mathrm{op}} \otimes_{\mathcal{O}_{X}} B\right)$ we conclude that ${ }_{A} \mathrm{~B}_{B} \in \operatorname{perf}\left(A^{\mathrm{op}} \otimes_{\mathcal{O}_{X}} B\right)$.

We now show the converse inclusion. Let ${ }_{A} \mathrm{~B}_{B}$ be an object of perf $\left(A^{\text {op }} \otimes_{\mathcal{O}_{X}}\right.$ $B) \subset \mathcal{D}\left(A^{\mathrm{op}} \otimes_{\mathcal{O}_{X}} B\right)$. Lemma 6.3 (with $A=A^{\mathrm{op}} \otimes_{\mathcal{O}_{X}} B$ ) shows us that $\mathrm{B} \in$ 
$\operatorname{perf}(X)$. Using again Lemma 6.3 (with $A=B$ ) we conclude that $\mathrm{B}_{B} \in \operatorname{perf}(B)$. By definition, this implies that ${ }_{A} \mathrm{~B}_{B} \in \operatorname{rep}(A, B)$ and so the proof if finished.

Let $\mathrm{Az}(X)$ be the category whose objects are the sheaves of Azumaya algebras over $X$, whose morphisms are given by $\operatorname{Hom}_{\mathrm{Az}(X)}(A, B):=\operatorname{Iso} \operatorname{rep}(A, B)$, and whose composition law is induced by

$$
\operatorname{rep}(A, B) \times \operatorname{rep}(B, C) \longrightarrow \operatorname{rep}(A, C) \quad\left({ }_{A} \mathrm{~B}_{B},{ }_{B} \mathrm{~B}_{C}^{\prime}\right) \mapsto{ }_{A} \mathrm{~B} \otimes_{B}^{\mathbb{L}} \mathrm{B}_{C}^{\prime} .
$$

Note that the identity of an object $A \in \mathrm{Az}(X)$ is given by the isomorphism class of the $A$-A-bimodule ${ }_{A} A_{A}$. The additivization of $\mathrm{Az}(X)$ is the additive category $\mathrm{Az}_{0}(X)$ with the same objects as $\mathrm{Az}(X)$ and with abelian groups of morphisms given by $\operatorname{Hom}_{\mathrm{Az}_{0}(X)}(A, B):=K_{0} \operatorname{rep}(A, B)$, where $K_{0} \operatorname{rep}(A, B)$ is the Grothendieck group of the triangulated category $\operatorname{rep}(A, B)$. The composition law is induced by the above bi-triangulated functor (8.2). Note that we have a functor

$$
\mathrm{Az}(X) \rightarrow \mathrm{Az}_{0}(X) \quad{ }_{A} \mathrm{~B}_{B} \mapsto\left[{ }_{A} \mathrm{~B}_{B}\right]
$$

Finally, the $\mathbb{Z}[1 / r]$-linearization of $\mathrm{Az}_{0}(X)$ is the $\mathbb{Z}[1 / r]$-linear category $\mathrm{Az}_{0}(X)_{1 / r}$ obtained by tensoring each abelian group of morphisms of $\mathrm{Az}_{0}(X)$ with $\mathbb{Z}[1 / r]$. This gives rise to the functor

$$
\mathrm{Az}_{0}(X) \rightarrow \mathrm{Az}_{0}(X)_{1 / r} \quad\left[{ }_{A} \mathrm{~B}_{B}\right] \mapsto\left[{ }_{A} \mathrm{~B}_{B}\right]_{1 / r}:=\left[{ }_{A} \mathrm{~B}_{B}\right] \otimes_{\mathbb{Z}} \mathbb{Z}[1 / r] .
$$

Proposition 8.3. Let $X, A$ be as in Theorem 2.1. Under these assumptions and the above notations, one has the isomorphism $\left[\mathcal{O}_{X} A_{A}\right]_{1 / r}: \mathcal{O}_{X} \stackrel{\sim}{\rightarrow} A$ in $\mathrm{Az}_{0}(X)_{1 / r}$.

Proof. By definition, $A$ is locally free of finite rank over $\mathcal{O}_{X}$. Consequently, the $A$ - $\mathcal{O}_{X}$-bimodule ${ }_{A} A_{\mathcal{O}_{X}}$ belongs to $\operatorname{rep}\left(A, \mathcal{O}_{X}\right)$ and so one obtains a well-defined morphism $\left[{ }_{A} A_{\mathcal{O}_{X}}\right]_{1 / r}: A \rightarrow \mathcal{O}_{X}$ in $\mathrm{Az}_{0}(X)_{1 / r}$. The proof will consist in showing that both compositions

$$
\left[\mathcal{O}_{X} A_{A}\right]_{1 / r} \circ\left[{ }_{A} A_{\mathcal{O}_{X}}\right]_{1 / r} \quad\left[{ }_{A} A_{\mathcal{O}_{X}}\right]_{1 / r} \circ\left[\mathcal{O}_{X} A_{A}\right]_{1 / r}
$$

are isomorphisms. Thanks to the above Lemma 8.1 (with $A=B=\mathcal{O}_{X}$ ), one has the following $\mathbb{Z}[1 / r]$-algebra isomorphism

$$
\operatorname{End}_{A z_{0}(X)_{1 / r}}\left(\mathcal{O}_{X}\right):=K_{0}\left(\operatorname{rep}\left(\mathcal{O}_{X}, \mathcal{O}_{X}\right)\right)_{1 / r} \simeq K_{0}(\operatorname{perf}(X))_{1 / r}=: K_{0}(X)_{1 / r}
$$

where the right-hand-side is endowed with the multiplication induced by $-\otimes_{\mathcal{O}_{X}}^{\mathbb{L}}-$. Since $A \otimes_{A} A \simeq A$, the composition $\left[\mathcal{O}_{X} A_{A}\right]_{1 / r} \circ\left[{ }_{A} A_{\mathcal{O}_{X}}\right]_{1 / r}$ equals $\left[\mathcal{O}_{X} A_{\mathcal{O}_{X}}\right]_{1 / r}$. Hence, since by hypothesis $A$ is of rank $\left(r_{1}, \ldots, r_{m}\right)$, we conclude from Corollary 2.4 and from Isomorphism (8.5) that $\left[\mathcal{O}_{X} A_{\mathcal{O}_{X}}\right]_{1 / r}$ is invertible in $\operatorname{End}_{\mathrm{Az}_{0}(X)_{1 / r}}\left(\mathcal{O}_{X}\right)$. The first composition in (8.4) is then an isomorphism.

Let us now prove that the second composition in (8.4) is also an isomorphism. Thanks to Lemma 8.1 (with $A=B$ ), one has the $\mathbb{Z}[1 / r]$-algebra isomorphism

$$
\operatorname{End}_{\mathrm{Az}_{0}(X)_{1 / r}}(A):=K_{0}(\operatorname{rep}(A, A))_{1 / r} \simeq K_{0}\left(\operatorname{perf}\left(A^{\mathrm{op}} \otimes_{\mathcal{O}_{X}} A\right)\right)_{1 / r},
$$

where the right-hand-side is endowed with the multiplication induced by $-\otimes_{A} \mathbb{L}_{-}$. On the other hand, Lemma 8.10 below furnishes us the following ring isomorphism

$$
K_{0}(X) \stackrel{\sim}{\longrightarrow} K_{0}\left(A^{\mathrm{op}} \otimes_{\mathcal{O}_{X}} A\right) \quad \mathcal{F} \mapsto \mathcal{F} \otimes_{\mathcal{O}_{X}}^{\mathbb{L}} A .
$$

Now, note that the composition $\left[{ }_{A} A_{\mathcal{O}_{X}}\right]_{1 / r} \circ\left[\mathcal{O}_{X} A_{A}\right]_{1 / r}$ is equal to $\left[{ }_{A} A \otimes \mathcal{O}_{X} A_{A}\right]_{1 / r}$. There exists then a unique element $\alpha$ in $K_{0}(X)$ which is mapped to $\left[{ }_{A} A \otimes_{\mathcal{O}_{X}} A_{A}\right]$ 
via the above isomorphism (8.7). We claim that $\operatorname{rank}(\alpha)=\left(r_{1}, \ldots, r_{m}\right)$. In order to prove this claim, consider the composed functor

$$
\operatorname{perf}(X) \stackrel{(8.11)}{\longrightarrow} \operatorname{perf}\left(A^{\text {op }} \otimes_{\mathcal{O}_{X}} A\right) \stackrel{\text { forget }}{\longrightarrow} \operatorname{perf}(X) .
$$

Since the $\mathcal{O}_{X}$-rank of $A$ is $\left(r_{1}, \ldots, r_{m}\right),(8.8)$ gives rise to the commutative square

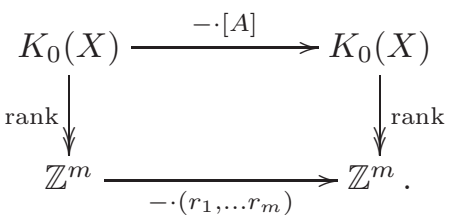

The equalities $\operatorname{rank}(\alpha \cdot[A])=\operatorname{rank}\left(\left[A \otimes_{\mathcal{O}_{X}} A\right]\right)=\left(r_{1}, \ldots, r_{m}\right)^{2}$, combined with the commutativity of (8.9) and the injectivity of the homomorphism $-\cdot\left(r_{1}, \ldots, r_{m}\right)$, allows us then to conclude that $\operatorname{rank}(\alpha)=\left(r_{1}, \ldots, r_{m}\right)$. Thanks to Corollary 2.4, the element $\alpha$ becomes then invertible in $K_{0}(X)_{1 / r}$ and so using (8.6) and the $\mathbb{Z}[1 / r]$-linearization of (8.7), one concludes that $\left[{ }_{A} A \otimes_{\mathcal{O}_{X}} A_{A}\right]_{1 / r}$ is invertible in $\operatorname{End}_{A z_{0}(X)_{1 / r}}(A)$. This implies that the second composition in (8.4) is also an isomorphism, and so the proof is finished.

Lemma 8.10. Let $X, A$ be as in Theorem 2.1. Under these assumptions, one has the following equivalence of monoidal triangulated categories

$$
\operatorname{perf}(X) \stackrel{\sim}{\longrightarrow} \operatorname{perf}\left(A^{\mathrm{op}} \otimes_{\mathcal{O}_{X}} A\right) \quad \mathcal{F} \mapsto \mathcal{F} \otimes_{\mathcal{O}_{X}}^{\mathbb{L}} A,
$$

where the monoidal structure on $\operatorname{perf}(X)$ (resp. on $\operatorname{perf}\left(A^{\mathrm{op}} \otimes_{\mathcal{O}_{X}} A\right)$ ) is induced by $-\otimes_{\mathcal{O}_{X}}^{\mathbb{L}}-\left(\right.$ resp. by $\left.-\otimes_{A}^{\mathbb{L}}-\right)$.

Remark 8.12. Since the monoidal structure on $\operatorname{perf}(X)$ is symmetric, we conclude from (8.11) that the monoidal structure on $\operatorname{perf}\left(A^{\mathrm{op}} \otimes_{\mathcal{O}_{X}} A\right)$ is also symmetric.

Proof. The fact that (8.11) is monoidal follows from the canonical isomorphisms

$$
\left(\mathcal{F} \otimes_{\mathcal{O}_{X}}^{\mathbb{L}} A\right) \otimes_{A}^{\mathbb{L}}\left(\mathcal{F}^{\prime} \otimes_{\mathcal{O}_{X}}^{\mathbb{L}} A\right) \simeq\left(\mathcal{F} \otimes_{\mathcal{O}_{X}}^{\mathbb{L}} \mathcal{F}^{\prime}\right) \otimes_{\mathcal{O}_{X}}^{\mathbb{L}} A .
$$

In order to prove that (8.11) is moreover an equivalence it suffices from Definition 6.1 to show the affine case where $X=\operatorname{Spec}(S)$ and $A$ is an Azumaya algebra over $S$. As explained in [26, §III Thm. 5.13$)]$, one has an equivalence of categories

$$
\operatorname{Mod}(S) \stackrel{\sim}{\longrightarrow} \operatorname{Mod}\left(A^{\mathrm{op}} \otimes_{S} A\right) \quad \mathcal{F} \mapsto \mathcal{F} \otimes_{S} A .
$$

Since (8.13) preserves finitely generated projective modules, one concludes from the definition of perfect complex that (8.11) is an equivalence in the affine case. This completes the proof.

Remark 8.14. Using Proposition 8.3, one observes that the category $A z_{0}(X)_{\mathbb{Q}}$ (obtained by tensoring each abelian group of morphisms of $\mathrm{Az}_{0}(X)$ with $\mathbb{Q}$ ) has a single isomorphism class. Kontsevich calls such categories "algebroids" [25, §1.1]. Intuitively speaking, all the complexity of $\mathrm{Az}_{0}(X)$ is torsion. 
From $\mathrm{Az}_{0}(X)$ to noncommutative motives. Let $A, B \in \mathrm{Az}_{0}(X)$. Note that every $A$ - $B$-bimodule ${ }_{A} \mathrm{~B}_{B} \in \operatorname{rep}(A, B)$ gives rise to a dg functor

$$
-\otimes_{A}^{\mathbb{L}} \mathrm{B}: \operatorname{perf}_{\mathrm{dg}}(A) \longrightarrow \operatorname{perf}_{\mathrm{dg}}(B) \quad \mathcal{F} \mapsto \mathcal{F}_{\text {flat }} \otimes_{A} \mathrm{~B}
$$

and consequently to a bimodule ${ }_{\left(-\otimes_{A}^{\mathbb{H}} \mathrm{B}\right)} \mathrm{Bi}$ which belongs to rep $\left(\operatorname{perf}_{\mathrm{dg}}(A), \operatorname{perf}_{\mathrm{dg}}(B)\right)$; recall from (4.2) the notation $-\mathrm{Bi}$. Similarly, every morphism $f:{ }_{A} \mathrm{~B}_{B} \rightarrow{ }_{A} \mathrm{~B}_{B}^{\prime}$ of $A$ - $B$-bimodules gives rise to a morphism of dg functors $\nu_{f}:-\otimes_{A}^{\mathbb{L}} \mathrm{B} \Rightarrow-\otimes_{A}^{\mathbb{L}} \mathrm{B}^{\prime}$ (see $[17, \S 2.3])$ and consequently to a morphism of bimodules ${ }_{\nu_{f}} \mathrm{Bi}:{ }_{-\otimes_{A}^{\mathbb{H}} \mathrm{B}} \mathrm{Bi} \Rightarrow{ }_{-\otimes_{A}^{\mathbb{L}} \mathrm{B}^{\prime}} \mathrm{Bi}$.

Lemma 8.15. The above constructions give rise to a triangulated functor

$$
\operatorname{rep}(A, B) \longrightarrow \operatorname{rep}\left(\operatorname{perf}_{\mathrm{dg}}(A), \operatorname{perf}_{\mathrm{dg}}(B)\right) .
$$

Proof. Note that when $f$ is a quasi-isomorphism, $\mathrm{H}^{0}\left(\nu_{f}\right)$ is a natural isomorphism between triangulated functors. Using [5, Lem. 9.8], one then concludes that ${ }_{\nu_{f}} \mathrm{Bi}$ is a quasi-isomorphism. This implies that (8.16) is well-defined. The fact that it is triangulated is clear.

Proposition 8.17. The assignment $A \mapsto U\left(\operatorname{perf}_{\mathrm{dg}}(A)\right)$ on objects and ${ }_{A} \mathrm{~B}_{B} \mapsto$ $U\left({ }_{\left(-\otimes_{A}^{\mathbb{L}} \mathrm{B}\right)} \mathrm{Bi}\right)$ on morphisms gives rise to a well-defined functor

$$
\mathrm{Az}_{0}(X) \longrightarrow \mathrm{Hmo}_{0}(k) \text {. }
$$

Proof. We start by verifying that the assignment $A \mapsto \operatorname{perf}_{\mathrm{dg}}(A)$ on objects and ${ }_{A} \mathrm{~B}_{B} \mapsto{ }_{\left(-\otimes_{A}^{\mathbb{L}} \mathrm{B}\right)} \mathrm{Bi}$ on morphisms gives rise to a well-defined functor from $\mathrm{Az}_{0}(X)$ to $\mathrm{Hmo}(k)$. Thanks to (8.16), one has well-defined morphisms

$$
\operatorname{Hom}_{\mathrm{Az}(X)}(A, B) \longrightarrow \operatorname{Hom}_{\mathrm{Hmo}(k)}\left(\operatorname{perf}_{\mathrm{dg}}(A), \operatorname{perf}_{\mathrm{dg}}(B)\right) \text {. }
$$

Given bimodules ${ }_{A} \mathrm{~B}_{B} \in \operatorname{rep}(A, B)$ and ${ }_{B} \mathrm{~B}_{C}^{\prime} \in \operatorname{rep}(B, C)$, the associativity of the (derived) tensor product gives rise to a canonical isomorphism of $\mathrm{dg}$ functors (and consequently to an isomorphism of bimodules)

$$
\left(-\otimes_{A}^{\mathbb{L}} \mathrm{B}\right) \otimes_{B}^{\mathbb{L}} \mathrm{B}^{\prime} \simeq-\otimes_{A}^{\mathbb{L}}\left(\mathrm{B} \otimes_{B}^{\mathbb{L}} \mathrm{B}^{\prime}\right) \quad\left(-\otimes_{A}^{\mathbb{L}} \mathrm{B}\right) \otimes_{B}^{\mathbb{L}} \mathrm{B}^{\prime} \mathrm{Bi} \simeq-_{-\otimes_{A}^{\mathbb{L}}\left(\mathrm{B} \otimes_{B}^{\mathbb{L}} \mathrm{B}^{\prime}\right)} \mathrm{Bi} .
$$

This shows that the assignment ${ }_{A} \mathrm{~B}_{B} \mapsto{ }_{\left(-\otimes_{A}^{\mathbb{L}} \mathrm{B}\right)} \mathrm{Bi}$ preserves the composition operation. The identities are also preserved since the $A$ - $A$-bimodule ${ }_{A} A_{A}$ is mapped to the identity bimodule id $\mathrm{Bi}=\operatorname{id}_{\operatorname{perf}_{\mathrm{dg}}(A)}$. In conclusion, we obtain a functor

$$
\mathrm{Az}(X) \longrightarrow \mathrm{Hmo}(k) \text {. }
$$

Now, from Lemma 8.15 and from the construction of the categories $\mathrm{Az}_{0}(X)$ and $\mathrm{Hmo}_{0}(k)$, one concludes that the searched functor (8.18) is the additivization of (8.19). This completes the proof.

We now have all the ingredients needed for the conclusion of the proof of Theorem 2.1. By $\mathbb{Z}[1 / r]$-linearizing the above functor (8.18) one obtains the following commutative diagram

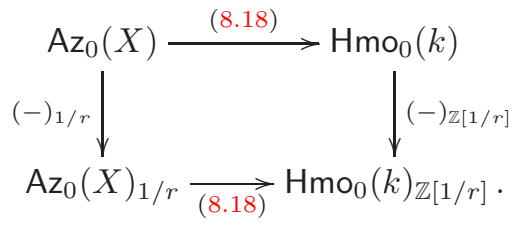


Hence, the image of the isomorphism $\left[\mathcal{O}_{X} A_{A}\right]_{1 / r}: \mathcal{O}_{X} \stackrel{\sim}{\rightarrow} A$ of Proposition 8.3 under (8.18) identifies with the following isomorphism

$$
U\left(-\otimes_{\mathcal{O}_{X}} A\right)_{\mathbb{Z}[1 / r]}: U\left(\operatorname{perf}_{\mathrm{dg}}(X)\right)_{\mathbb{Z}[1 / r]} \stackrel{\sim}{\longrightarrow} U\left(\operatorname{perf}_{\mathrm{dg}}(A)\right)_{\mathbb{Z}[1 / r]} .
$$

This completes the proof.

\section{Proof of Theorem 3.13}

Let us write $\pi: S \rightarrow S / I$ for the quotient map. One needs to show that it yields an isomorphism $U(\underline{\pi})_{R}: U(\underline{S})_{R} \stackrel{\sim}{\rightarrow} U(S / I)_{R}$. By the Yoneda lemma for the full subcategory of $\mathrm{Hmo}_{0}(k)_{R}$ containing the objects $U(\underline{S})_{R}$ and $U(S / I)_{R}$, one observes that it suffices to show that the induced homomorphism

$$
\left(U(\underline{\pi})_{R}\right)_{*}: \operatorname{Hom}_{\mathrm{Hmo}_{0}(k)_{R}}\left(U(\underline{T})_{R}, U(\underline{S})_{R}\right) \longrightarrow \operatorname{Hom}_{\mathrm{Hmo}_{0}(k)_{R}}\left(U(\underline{T})_{R}, U(\underline{S / I})_{R}\right)
$$

is an isomorphism for $T=S, S / I$. Concretely, is suffices to show that

$$
\left[-\otimes_{S}^{\mathbb{L}} \pi \mathrm{Bi}\right]: K_{0}(\operatorname{rep}(\underline{T}, \underline{S}))_{R} \longrightarrow K_{0}(\operatorname{rep}(\underline{T}, \underline{S / I}))_{R}
$$

is an isomorphism. The proof is now divided into two cases.

Case 1. $(S / J(S) k$-separable) Assume that $S / I$ has finite global dimension and that $S / J(S)$ is $k$-separable. Since $(S / I) / J(S / I)=S / J(S)$, one concludes then form [10, page 2] that the dg categories $\underline{S}$ and $\underline{S} / I$ are smooth. They are also proper, and so thanks to description (5.5) the induced homomorphism (9.1) reduces to

$$
\left[-\otimes_{S}^{\mathbb{L}} \pi \mathrm{Bi}\right]: K_{0}\left(T^{\mathrm{op}} \otimes S\right)_{R} \longrightarrow K_{0}\left(T^{\mathrm{op}} \otimes S / I\right)_{R} .
$$

Now, recall that by assumption $I$ is nilpotent. As a consequence, the (two-sided) ideal of the quotient map $T^{\mathrm{op}} \otimes S \rightarrow T^{\mathrm{op}} \otimes(S / I)$ is also nilpotent. Using the invariance of the Grothendieck group functor with respect to nilpotent extensions (see [42, page 70]), we hence conclude that (9.2) is an isomorphism.

Case 2. $(1 / p \in R)$ Assume that $S / I$ has finite global dimension and that $k$ is a field of characteristic $p>0$ such that $1 / p \in R$. Note that since $S$ and $S / I$ are finite dimensional and of finite global dimension we have the equivalences

$$
\operatorname{rep}(\underline{T}, \underline{S}) \simeq \mathcal{D}^{b}\left(\bmod \left(T^{\mathrm{op}} \otimes S\right)\right) \quad \operatorname{rep}(\underline{T}, \underline{S / I}) \simeq \mathcal{D}^{b}\left(\bmod \left(T^{\mathrm{op}} \otimes S / I\right)\right),
$$

where $\mathcal{D}^{b}(\bmod (-))$ stands for the bounded derived category of finitely generated modules. The above homomorphism (9.1) identifies then with

$$
\left[-\otimes_{S}^{\mathbb{L}} \pi \mathrm{Bi}\right]: G_{0}\left(T^{\mathrm{op}} \otimes S\right)_{R} \longrightarrow G_{0}\left(T^{\mathrm{op}} \otimes S / I\right)_{R} .
$$

Now, consider the following quotient maps

$$
r_{S}: S \rightarrow S / J(S) \quad q_{T}: T \rightarrow T / J(T) \quad q_{S / I}: S / I \rightarrow S / J(S / I)=S / J(S) .
$$


Using the following commutative diagram ${ }^{5}$

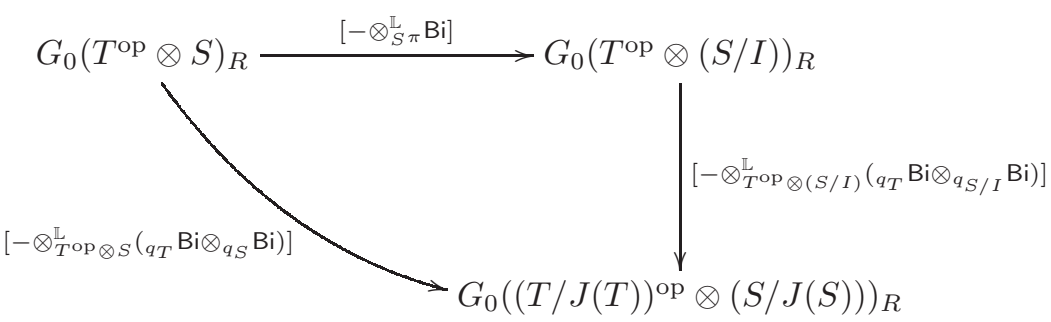

one observes that it suffices to prove that

$$
\left[-\otimes_{T^{\mathrm{op}} \otimes S}^{\mathbb{L}}\left(q_{T} \mathrm{Bi} \otimes_{q_{S}} \mathrm{Bi}\right)\right]: G_{0}\left(T^{\mathrm{op}} \otimes S\right)_{R} \longrightarrow G_{0}\left((T / J(T))^{\mathrm{op}} \otimes(S / J(S))\right)_{R}
$$

is an isomorphism. Moreover, it is sufficient by base-change to treat the case $R=$ $\mathbb{Z}[1 / p]$. Note that the kernel of the quotient map $q_{T}^{\text {op }} \otimes q_{S}$ is nilpotent. Hence, $G_{0}\left(T^{\mathrm{op}} \otimes S\right)$ and $G_{0}\left((T / J(T))^{\mathrm{op}} \otimes(S / J(S))\right.$ are free $\mathbb{Z}$-modules with a basis given by the simple $\left((T / J(T))^{\text {op }} \otimes(S / J(S))\right.$-modules. In particular, they have the same rank. As a consequence, it suffices to prove that (9.3) (with $R=\mathbb{Z}[1 / p]$ ) is a surjection. In order to do so, we consider the following commutative diagram

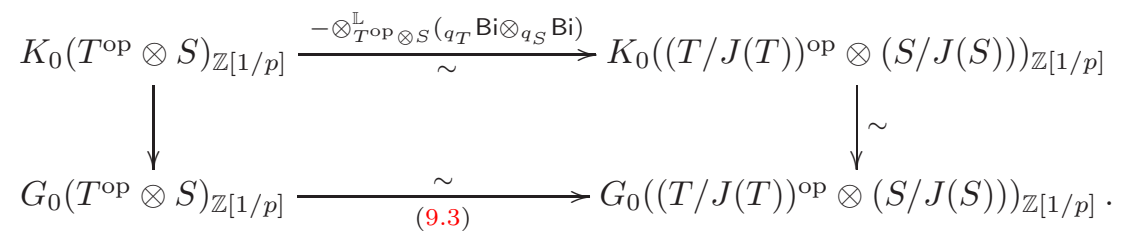

As in the proof of Case 1, the upper horizontal map is an isomorphism. Thanks to Proposition 9.4 below (with $U=T / J(T)$ and $U^{\prime}=S / J(S)$ ) the right vertical map is also an isomorphism. Using these isomorphisms and the commutativity of the above diagram we conclude that (9.3) is a surjection. This finishes the proof.

Proposition 9.4. Given a field $k$ of characteristic $p>0$, the induced map

$$
K_{0}\left(U \otimes U^{\prime}\right)_{\mathbb{Z}[1 / p]} \longrightarrow G_{0}\left(U \otimes U^{\prime}\right)_{\mathbb{Z}[1 / p]}
$$

is an isomorphism for any two finite dimensional semi-simple $k$-algebras $U$ and $U^{\prime}$.

Proof. Since $U \otimes U^{\prime}$ is finite dimensional, $K_{0}\left(U \otimes U^{\prime}\right)_{\mathbb{Z}[1 / p]}$ and $G_{0}\left(U \otimes U^{\prime}\right)_{\mathbb{Z}[1 / p]}$ are free $\mathbb{Z}$-modules with the same rank. Hence, it suffices to show that (9.5) is surjective. One can (and will) assume without loss of generality that $U$ and $U^{\prime}$ are indecomposable. Let $Z$ (resp. $Z^{\prime}$ ) be the center of $U$ (resp. of $U^{\prime}$ ) and $Z_{0}$ (resp. $Z_{0}^{\prime}$ ) the separable closure of $k$ in $Z$ (resp. in $Z^{\prime}$ ). Under these notations, one has $Z_{0} \otimes Z_{0}^{\prime}=\bigoplus_{i} W_{i}$ with $W_{i} / k$ a separable field extension. As a consequence, one obtains the following equalities:

$$
\begin{aligned}
U \otimes U^{\prime} & =U \otimes_{Z_{0}}\left(Z_{0} \otimes Z_{0}^{\prime}\right) \otimes_{Z_{0}^{\prime}} U^{\prime} \\
& =\bigoplus_{i}\left(U \otimes_{Z_{0}} W_{i} \otimes_{Z_{0}^{\prime}} U^{\prime}\right) \\
& =\bigoplus_{i}\left(U \otimes_{Z_{0}^{\prime}} W_{i}\right) \otimes_{W_{i}}\left(U^{\prime} \otimes_{W_{0}} W_{i}\right) .
\end{aligned}
$$

\footnotetext{
${ }^{5}$ Note that although $T^{\mathrm{op}} \otimes S$ may have infinite global dimension, it is still true that $(T / J(T))^{\mathrm{op}} \otimes(S / J(S))$ has finite projective dimension over $T^{\mathrm{op}} \otimes S$.
} 
Replacing $k$ by $W_{i}$ and $U$ (resp. $U^{\prime}$ ) by $U \otimes_{Z_{0}} W_{i}$ (resp. by $U^{\prime} \otimes_{Z_{0}^{\prime}} W_{i}$ ) one can (and will) assume that $Z$ and $Z^{\prime}$ are purely inseparable $k$-algebras. We hence have

$$
U \otimes U^{\prime}=\left(U \otimes Z^{\prime}\right) \otimes_{\left(Z \otimes Z^{\prime}\right)}\left(U^{\prime} \otimes Z^{\prime}\right)
$$

Note that $D:=U \otimes U^{\prime}$ is the tensor product of two Azumaya algebras over $W:=$ $Z \otimes Z^{\prime}$, and hence is itself and Azumaya algebra. Thanks to Lemma 9.6 below, $W$ is a local $k$-algebra. By lifting idempotents and invoking Morita equivalence the problem of showing that (9.5) is surjective can be reduced to the case that $D / J(D)$ is a division algebra. In this case, $D / J(D)=W / J(W) \otimes_{W} D$ is the unique simple $D$-module. Invoking Lemma 9.6 again, we find that $D=W \otimes_{W} D$ is an extension of $p^{n}$ copies (for some $n$ ) of $W / J(W) \otimes_{W} D$. Hence, $p^{n}[D / J(D)]$ is in the image of (9.5). This finishes the proof.

Lemma 9.6. Let $k$ be a field of characteristic $p>0$ and $Z / k, Z^{\prime} / k$ two purely inseparable field extensions. Under these assumptions, $Z \otimes Z^{\prime}$ is a local $k$-algebra and its length (as a module over itself) is a power of $p$.

Proof. Note that if $e \in Z \otimes Z^{\prime}$, then $e^{p^{n}} \in k$ for some $n \gg 0$. In the case where $e$ is an idempotent we then conclude that $e=0,1$. This implies that $Z \otimes Z^{\prime}$ is a local $k$-algebra. It is also clear that the length of $Z \otimes Z^{\prime}$ must $\operatorname{divide} \operatorname{dim}_{k}\left(Z \otimes Z^{\prime}\right)$. Hence, it is necessarily a power of $p$.

\section{REFERENCES}

[1] B. Antieau, Letter to the authors. July 31, 2013.

[2] M. Auslander and O. Goldman, The Brauer group of a commutative ring. Trans. Amer. Math. Soc. 97 (1960), 367-409.

[3] Goro Azumaya, On maximally central algebras. Nagoya Math. J. 2 (1951), 119-150.

[4] M. Bernardara and G. Tabuada, Motivic decompositions in the commutative and noncommutative world. Available at arXiv:1303.3172.

[5] _ From semi-orthogonal decompositions to polarized intermediate Jacobians via Jacobians of noncommutative motives. Available at arXiv:1305.4687.

[6] R. Bezrukavnikov, I. Mirković and D. Rumynin, Localization of modules for a semisimple Lie algebra in prime characteristic. Annals of Mathematics 167 (2008), 945-991.

[7] A. Blumberg and M. Mandell, Localization theorems in topological Hochschild homology and topological cyclic homology. Geometry and Topology 16 (2012), 1053-1120.

[8] A. Bondal and D. Orlov, Semiorthogonal decomposition for algebraic varieties. Available at arXiv:alg-geom/9506012.

[9] A. Bondal and M. Van den Bergh, Generators and representability of functors in commutative and noncommutative geometry. Mosc. Math. J. 3, no. 1, (2003), 1-36.

[10] R. Buchweitz, E. Green, D. Madsen, and Ø. Solberg, Finite Hochschild cohomology without finite global dimension. Math. Res. Lett. 12 (2005), no. 5-6, 805-816.

[11] D.-C. Cisinski and G. Tabuada, Symmetric monoidal structure on noncommutative motives. Journal of $K$-Theory 9 (2012), no. 2, 201-268.

[12] G. Cortiñas and C. Weibel, Homology of Azumaya algebras. Proceedings of the American Mathematical Society 121 (1994), no. 1, 53-55.

[13] W. Fulton and S. Lang, Riemann-Roch algebra. Grundlehren der Mathematischen Wissenschaften 277. Springer-Verlag, New York, 1985.

[14] O. Gabber, Some theorems on Azumaya algebras. The Brauer group, 129-209, Lecture Notes in Math. 844, Springer, Berlin-New York, 1981.

[15] A. Grothendieck, Le groupe de Brauer I: Algèbres d'Azumaya et interprétations diverses. Dix Exposés sur la Cohomologie des Schémas, 46-66. North-Holland, Amsterdam; Masson, Paris.

[16] R. Hazrat and R. T. Hoobler, K-theory of Azumaya algebras over schemes. Communications in Algebra 41 (2013), no. 4, 1268-1277. 
[17] B. Keller, On differential graded categories. International Congress of Mathematicians (Madrid), Vol. II, 151-190. Eur. Math. Soc., Zürich (2006).

[18] - On the cyclic homology of exact categories. Journal of Pure and Applied Algebra 136 (1999), no. 1, 1-56.

[19] - On the cyclic homology of ringed spaces and schemes. Doc. Math. 3 (1998), 231-259.

[20] Invariance and localization for cyclic homology of dg algebras. Journal of Pure and Applied Algebra 123 (1998), no. 1-3, 223-273.

[21] M. Kontsevich, Noncommutative motives. Talk at the Institute for Advanced Study on the occasion of the $61^{\text {st }}$ birthday of Pierre Deligne, October 2005. Video available at http://video.ias.edu/Geometry-and-Arithmetic.

[22] — Triangulated categories and geometry. Course at the École Normale Supérieure, Paris, 1998. Notes available at www.math.uchicago.edu/mitya/langlands.html

[23] _ Mixed noncommutative motives. Talk at the Workshop on Homological Mirror Symmetry. University of Miami. 2010. Notes available at ww-math.mit.edu/auroux/frg/miami10-notes.

[24] - Notes on motives in finite characteristic. Algebra, arithmetic, and geometry: in honor of Yu. I. Manin. Vol. II, 213-247, Progr. Math., 270, Birkhuser Boston, MA, 2009.

[25] _ Deformation quantization of algebraic varieties, Lett. Math. Phys. 56 (2001), no. 3, 271-294. EuroConférence Moshé Flato 2000, Part III (Dijon).

[26] M.-A. Knus and M. Ojanguren, Théorie de la descente et algèbres d'Azumaya. Lecture Notes in Mathematics 389. Springer-Verlag, Berlin-New York (1974).

[27] A. Kuznetsov, Derived Categories of Cubic Fourfolds. Cohomological and Geometric Approaches to Rationality Problems. New Perspectives Series: Progress in Mathematics, Vol. 282, Bogomolov, Fedor; Tschinkel, Yuri (Eds.) 2010.

[28] T. Y. Lam, Introduction to Quadratic forms over fields. Graduate Studies in Mathematics 67. American Mathematical Society.

[29] V. Lunts and D. Orlov, Uniqueness of enhancement for triangulated categories. J. Amer. Math. Soc. 23 (2010), 853-908.

[30] M. Marcolli and G. Tabuada, From exceptional collections to motivic decompositions via noncommutative motives. Available at arXiv:1202.6297. To appear in Journal für die reine und angewandte Mathematik (Crelle).

[31] D. Quillen, Higher algebraic K-theory: I. Higher K-theories (Proc. Conf., Battelle Memorial Inst., Seattle, Wash., 1972), pp. 85-147. Lecture Notes in Math. 341, Springer, Berlin 1973.

[32] P. Revoy, Algèbres de Weyl en caractéristique p. C.R. Acad. Sci. Paris. Sér. A-B 276 (1973), A $225-228$.

[33] M. Schlichting, Negative K-theory of derived categories. Math. Z. 253 (2006), no. 1, 97-134.

[34] G. Tabuada, A guided tour through the garden of noncommutative motives. Clay Mathematics Proceedings, Volume 16 (2012), 259-276.

[35] _ Generalized spectral categories, topological Hochschild homology, and trace maps. Algebraic and Geometric Topology 10 (2010), 137-213.

[36] _ Higher K-theory via universal invariants. Duke Math. J. 145 (2008), no. 1, 121-206.

[37] _ Additive invariants of dg categories. Int. Math. Res. Not. 53 (2005), 3309-3339.

[38] R. W. Thomason, The classification of triangulated subcategories. Compositio Mathematica 105, no. 1, (1997), 1-27.

[39] R. W. Thomason and T. Trobaugh, Higher algebraic K-theory of schemes and of derived categories. Grothendieck Festschrift, Volume III. Volume 88 of Progress in Math., 247-436. Birkhauser, Boston, Bassel, Berlin, 1990.

[40] B. Toën, Derived Azumaya algebras and genertors for twisted derived categories. Invent. Math. 189 (2012), no. 3, 581-652.

[41] F. Waldhausen, Algebraic K-theory of spaces. Algebraic and Geometric topology (New Brunswick, N. J., 1983), 318-419, Lecture Notes in Math., 1126, Springer, Berlin, 1985.

[42] C. Weibel, K-book. Available at http://www.math.rutgers.edu/ $\sim$ weibel/Kbook.pdf.

Gonçalo Tabuada, Department of Mathematics, mit, Cambridge, Ma 02139, USA

E-mail address: tabuada@math.mit.edu

URL: http://math.mit.edu/ tabuada 
Michel Van den Bergh, Departement Wni, Universiteit Hasselt, 3590 Diepenbeek, BELGIUM

E-mail address: michel.vandenbergh@uhasselt.be

$U R L$ : http://hardy. uhasselt.be/personal/vdbergh/Members/ michelid.html 